\title{
Inter-Disciplinary Collaboration in Support of the Post-Standby TREAT Mission
}

\author{
Mark DeHart ${ }^{1}$, Benjamin Baker ${ }^{1}$, Javier Ortensi ${ }^{1}$, \\ Nicolas Woolstenhulme ${ }^{1}$, John Bess ${ }^{1}$, Colby Jensen ${ }^{1}$, \\ James Parry ${ }^{1}$, Tony Hill ${ }^{2}$, William Phoenix ${ }^{3}$ \\ ${ }^{1}$ Idaho National Laboratory \\ 2525 Fremont St \\ Idaho Falls, ID 83415 \\ ${ }^{2}$ Idaho State University \\ 921 S 8th Ave \\ Pocatello, ID 83209 \\ ${ }^{3}$ Walsh Engineering Services PC \\ 330 Shoup Ave \# 300 \\ Idaho Falls, ID 83402
}

September 2016

INL is a U.S. Department of Energy National Laboratory operated by Battelle Energy Alliance 


\section{DISCLAIMER}

This information was prepared as an account of work sponsored by an agency of the U.S. Government. Neither the U.S. Government nor any agency thereof, nor any of their employees, makes any warranty, expressed or implied, or assumes any legal liability or responsibility for the accuracy, completeness, or usefulness, of any information, apparatus, product, or process disclosed, or represents that its use would not infringe privately owned rights. References herein to any specific commercial product, process, or service by trade name, trade mark, manufacturer, or otherwise, does not necessarily constitute or imply its endorsement, recommendation, or favoring by the U.S. Government or any agency thereof. The views and opinions of authors expressed herein do not necessarily state or reflect those of the U.S. Government or any agency thereof. 


\section{Inter-Disciplinary Collaboration in Support of the Post-Standby TREAT Mission}

Mark DeHart (INL), Benjamin Baker (INL), Javier Ortensi (INL), Nicolas Woolstenhulme (INL), John Bess INL), Colby Jensen (INL), James Parry (INL), Tony Hill (ISU), William Phoenix (Walsh)

September 2016

Idaho National Laboratory

Nuclear Systems Design and Analysis

Idaho Falls, Idaho 83415

http://www.inl.gov

Prepared for the

U.S. Department of Energy

Office of Nuclear Energy

Under DOE Idaho Operations Office

Contract DE-AC07-05ID14517 


\section{Contents}

1 Introduction 1

2 Advanced Modeling and Simulation 3

3 Stakeholder Needs $\quad 7$

3.1 Operational Needs . . . . . . . . . . . . . . . . 7

3.2 Experiment Design and Experimentalist's Needs . . . . . . . . . . . . . . 9

3.3 Instrumentation Needs . . . . . . . . . . . . . . . . . . 11

3.4 Benchmark Development Needs . . . . . . . . . . . . . . . 12

3.5 MAMMOTH Modeling and Simulations Needs . . . . . . . . . . . . 13

3.5.1 Reproduction of the M8 Calibration Tests . . . . . . . . . . . . . . 14

3.5.2 Multi-SERTTA Calibration . . . . . . . . . . . . . . 14

3.5.3 TREAT Temperature Measurements . . . . . . . . . . . . 15

3.5.4 TREAT Flux Measurements . . . . . . . . . . . . . . . . . 15

3.5.5 Characterize Transient Capabilities in TREAT . . . . . . . . . . . 16

3.5.6 Neutron Lifetime and Beta Measurements . . . . . . . . . . . . . 16

3.5.7 Neutron Spectrum Characterization . . . . . . . . . . . . . 16

4 Advanced Modeling for the Multi-SERTTA Irradiation Vehicle 17

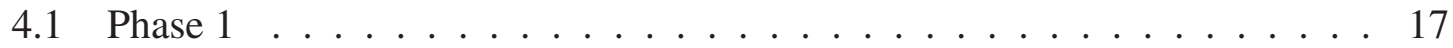

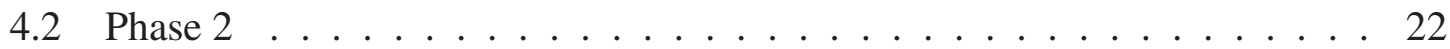

5 Design and Modeling of Micro-Pocket Fission Detectors 25

6 Modeling and Simulation Support 29

6.1 Reactivity Computer . . . . . . . . . . . . . . . . . 29

6.2 Flux and Power Estimations . . . . . . . . . . . . . . . 30

6.3 Multi-SERTTA Modeling with MAMMOTH . . . . . . . . . . . . 32

6.3.1 Cross Section Preparation . . . . . . . . . . . . . . 32

6.3 .2 Mesh Development . . . . . . . . . . . . . 36

6.3 .3 Results ...................... 37

6.3.4 Next Steps in Support of Transient Simulations . . . . . . . . . . 46

$\begin{array}{lll}7 & \text { Conclusions } & 47\end{array}$ 


\section{List of Figures}

1 Parallel Timelines for TREAT Restart, Modeling, and Validation . . . . . 4

2 The ideal experiment is an evolving target based on iterative exchanges between experiments and modeling. . . . . . . . . . . . . . 10

3 ATF instrument array within a SERTTA capsule. . . . . . . . . . . . 12

4 Example core map (left), package type vehicle in TREAT core section view (right). . . . . . . . . . . . . . . . . . . . . . 18

5 Multi-SERTTA Full Assembly . . . . . . . . . . . . . . . . . . . . . . . . 19

6 Rod Position Cases . . . . . . . . . . . . . . . . . . . . . . . 21

7 Multi-SERTTA and included MPFD assembly will be deployed within the central test channel at TREAT. . . . . . . . . . . . . . . . . . 26

8 Illustration of the most current micro-pocket fission detector design $\ldots .27$

9 Reactivity Computer Simulator Layout . . . . . . . . . . . . . . . . . 30

10 Serpent Model of the SERTTA Unit . . . . . . . . . . . . . . . . 33

11 Serpent Model of the $5 \times 5$ Supercell with the SERTTA Unit . . . . . . . 34

12 Full Core Serpent Model with the Multi-SERTTA Vehicle. . . . . . . . . . 35

13 Pin Radial and Azimuthal Regions with Material Identification . . . . . . 36

14 Extruded (left) and Hybrid (right) Meshes for the $5 \times 5$ Supercell . . . . . . 37

15 Flux Distributions in the $5 \times 5$ SERTTA Supercell . . . . . . . . . . . . 40

16 Flux Distributions in a Slice of the $5 \times 5$ SERTTA Supercell $\ldots . . . .44$

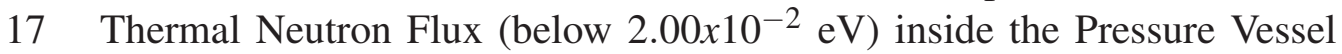
and Fuel Pin . . . . . . . . . . . . . . . . . . . . . . . 42

18 Fast Flux (above $3.33 \times 10^{6} \mathrm{eV}$ ) in the Fuel Pellet Stack (clip at axial centerline) . . . . . . . . . . . . . . . . . . . . . 43

19 Thermal Flux (8.10 to $0.625 \mathrm{eV}$ ) in the Fuel Pellet Stack (clip at axial

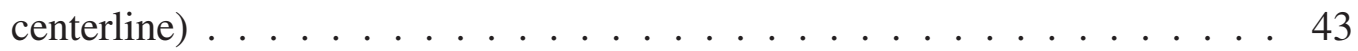

20 Thermal Flux (below $2.00 \times 10^{-2} \mathrm{eV}$ ) in the Fuel Pellet Stack (clip at axial centerline $\ldots \ldots \ldots \ldots \ldots \ldots$. . . . . . . . . . . . 44

21 Power Density [W/cc] in the Fuel Pellet Stack (clip at axial centerline) . . . 44

22 Diametric Power Density Profile at the Centerline (south to north) . . . . 45 


\section{List of Tables}

1 Values of $k_{\text {eff }}$ Calculated for the $5 \times 5$ SERTTA Supercell $\ldots . . . . .38$

2 Percentage Difference in Integral Parameters for the $5 \times 5$ SERTTA Supercell Relative to the Serpent Reference Solution . . . . . . . . . . . . 38

3 Percentage Difference in Pellet Powers for the $5 \times 5$ SERTTA Supercell

Relative to the Serpent Reference Solution . . . . . . . . . . . . . . . 38 


\section{Introduction}

The Transient Reactor Test Facility (TREAT) is an air-cooled, graphite moderated, thermal spectrum test nuclear reactor designed to test reactor fuels and structural materials [1]. Constructed in 1958, and operated from 1959 until 1994, TREAT was built to perform transient reactor experiments in which a test material, positioned in the center of the reactor core, is subjected to high energy neutron pulses that can be tailored to simulate off-normal reactor conditions ranging from mild transients to severe accidents. The reactor was placed into operational standby status in 1994.

The U.S. Department of Energy has decided to resume a program of transient testing [2] and plans to restart TREAT facility by 2018. The renewed interest in TREAT was sparked by the 2011 Fukushima Daiichi nuclear disaster, which prompted the shutdown of nuclear plants in Japan and Germany. Currenly the first panned use of TREAT is to support testing of new accident tolerant fuel concepts for nuclear reactors [3].

TREAT is a test facility designed to evaluate reactor fuels and structural materials under conditions that simulate various types of transient overpower and under-cooling situations in a nuclear reactor, but it can do more. TREAT contributions to reactor safety programs have been threefold: (1) to provide basic data for predicting the safety margin of fuel designs and the severity of potential accidents, (2) to serve as a proving ground for fuel concepts design to reduce or preclude the consequent hazards associated with potential accidents, and (3) to provide non-destructive test data through neutron radiography of fuel samples. The resumption of TREAT operations resurrects the requisite transient engineering platform for qualifying new accident tolerant fuel forms but there are also unique opportunities to extend the mission space of this system in support of higher resolution, lower length-scale phenomena through focused efforts between experimentalists, theorists, modelers and code developers.

Historic transient testing in TREAT relied upon an extensive series of transient and steadystate experiments typically irradiating wires and some fuel pins to envelope the desired region of applicability in which the final experiment would be targeted. Then a final set of three experiment tests were performed in the calibration vehicle for fine-tuning of the actual transient test experiment to be performed. Basic point kinetics modeling and neutronics core analyses were performed, but mostly to verify, on a limited basis, what was already estimated experimentally. Dozens of supporting experiments could have been performed to estimate the desired test configuration within a given test series. 
The performance of multiple experiment runs has always been a time-consuming and expensive process, but much more accurate and timely than the computational modeling capabilities historically available for transient testing. Advanced modeling and simulation cannot fully replace experimental testing, but can be utilized to significantly reduce the number of experimental tests that would need to be performed in future TREAT transient tests. The mission-driven needs of the reactor can, and will, be significantly enhanced through advanced modeling and simulation capabilities as unique experiment designs require a more thorough understanding of the 3-dimensional nature of TREAT reactor kinetics and performance. 


\section{Advanced Modeling and Simulation}

Although analysis methods have advanced significantly in the last two decades, high fidelity multi-physics methods for reactors systems have been under development for only a few years and are not presently mature nor deployed. Furthermore, very few methods provide the ability to simulate rapid transients in three dimensions. Data for validation of advanced time-dependent multi-physics is sparse; at TREAT, historical data were not collected for the purpose of validating three-dimensional methods, let alone multi-physics simulations. Existing data continues to be collected to attempt to simulate the behavior of experiments and calibration transients $[4,5,6]$, but it will be insufficient for the complete validation of analysis methods used for TREAT transient simulations. Hence, a 2018 restart will most likely occur without the direct application of advanced modeling and simulation (M\&S) methods. At present, the current INL modeling and simulation team plans to work with TREAT operations staff in performing reactor simulations with MAMMOTH [7], in parallel with the software packages currently being used in preparation for core restart (e.g., MCNP5 [8], RELAP5 [9], ABAQUS [10]). The TREAT team has also requested specific measurements to be performed during startup testing, currently scheduled to run from February to August of 2018. The current timeline for startup measurements and testing, along with modeling efforts and validation, is shown in Fig. 1. These startup measurements will be crucial in validating the new analysis methods in preparation for ultimate application for TREAT operations and experiment design.

Even though TREAT will perform startup testing based on lower order transient simulations, there will be limits to how much can be achieved using such methods. Technology has evolved significantly in the 22 years since the last transient test performed at TREAT. High-fidelity multi-physics methods will provide opportunities to expand the scope of TREAT experiments and advance the state-of-the-art of fuels development programs. The nature of materials research has also changed considerably, from an engineering and scale testing approach to a science-based, engineering driven paradigm with a heavy reliance on the technological developments of the last two decades. The revolutionary predictive power sought for rapid fuels development will require seamless integration of multi-physics analysis to begin to approach the intimately coupled interactions that drive material changes under irradiation. But such analysis capabilities cannot stand alone. They will require development of novel experimentation techniques in parallel with, and cognizant of, advances in the methodologies of computational materials science and high-fidelity simulations. The importance of identifying and performing experiments of highest impact cannot be overstated. The scope of the TREAT research program can be extended into smaller length and time scales by integrating appropriately evolved instrumentation and computational devel- 

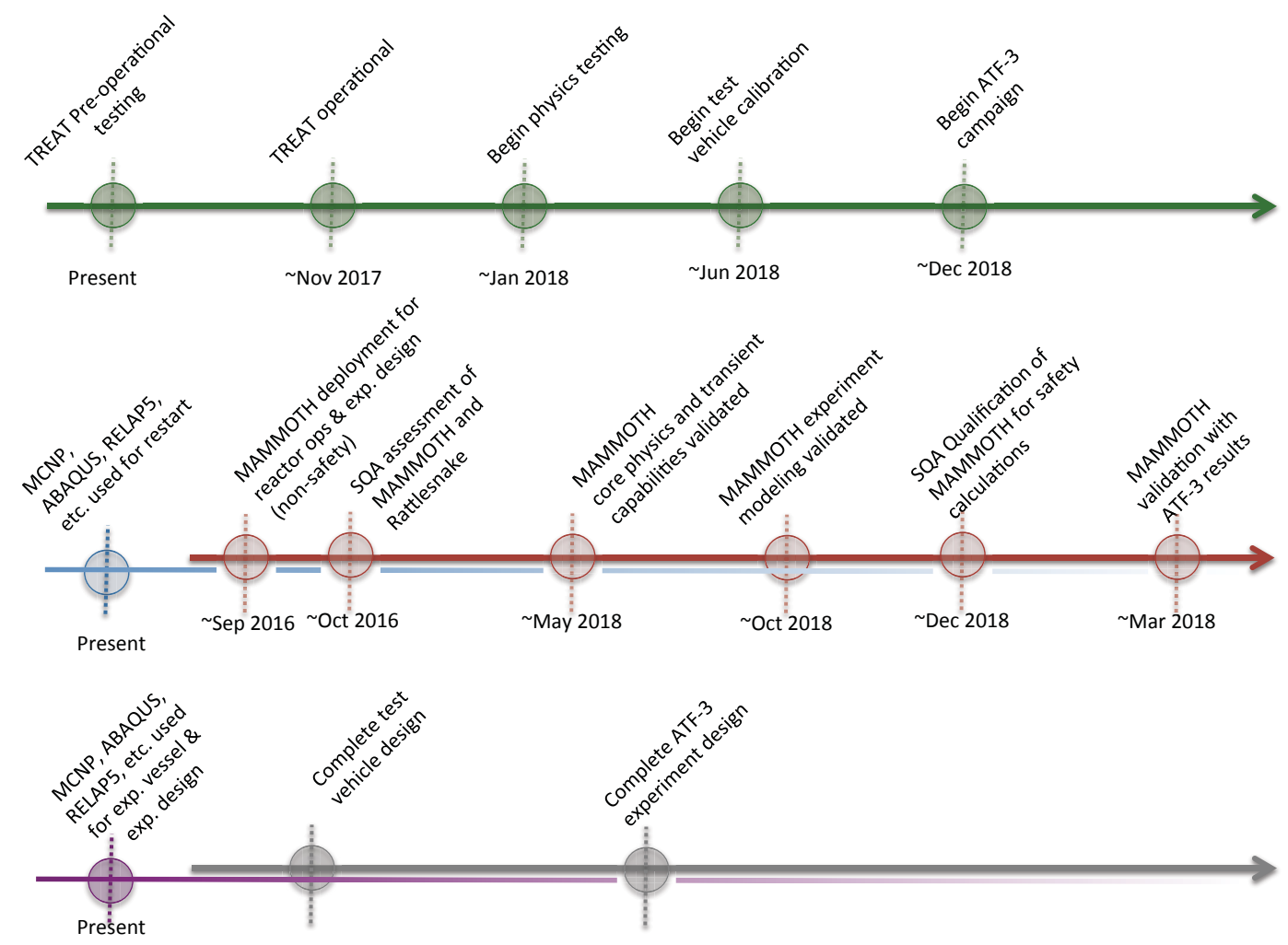

Figure 1: Parallel Timelines for TREAT Restart, Modeling, and Validation

opments for simulating, analyzing and mining what will be vast amounts of experimental and simulated information.

The MOOSE framework [11] has been developed in response to the need for developing a multi-scale, multi-physics simulation capability that spans from basic first principles to the engineering scale, including nuclear physics, materials, thermal fluids, mathematics, and nuclear, mechanical, civil, and chemical engineering, all linked together with computer science. The effort has been widely recognized, culminating in an R\&D 100 Award in 2014. MOOSE is designed to accelerate research and scientific understanding and provides a perfect backbone for the integration of experiments and simulations. Supported by the DOE Nuclear Energy Advanced Modeling and Simulation program (NEAMS), the MOOSEbased MAMMOTH reactor physics application has already been adopted for use in the TREAT program and applied to an increasingly complicated set of TREAT simulations 
$[12,13]$. Ongoing work has focused on the development of cross section treatments to improve the efficiency and accuracy of TREAT calculations [14], along with development of a full-core model of the current core, for simulation of the last series of experiment calibrations, the M8-CAL series [15]. At the same time, the MAMMOTH team has been working to optimize strongly coupled thermal-hydraulic, neutronic and fuels performance calculations using RELAP-7 [16], Rattlesnake [17] and BISON [18] within MAMMOTH, to be able to simulate the physics of experiment configurations [19].The ultimate goal is to extend the capabilities of MAMMOTH to support the development, design and analysis of experiments, at all length scales, using appropriate simulations.

There are no true separate effects experiments when neutron irradiation is involved. MAMMOTH is key to the evolution of novel TREAT experimental designs. Even the simplest TREAT irradiation experiments are a convolution of several phenomena over varying time and length scales. Identifying and designing the best experiments, or sets of experiments, will result in less redundant and more informative data, maximizing the relevance and impact of any proposed experimental effort. High-fidelity simulations that may be used for accelerating experiment design evolution is also of high value, providing the necessary embedded tools to perform trade-off studies during experiment design and development. This activity will certainly require a close collaboration between experimentalists and modelers; in fact, as will be discussed later, such collaborations are already underway. Based on the current ongoing interactions, it is expected that MAMMOTH could and should be used to develop a comprehensive management portfolio for all TREAT upgrade investments and experiments.

Clearly there is a need for TREAT staff, experiment designers, modelers and methods developers at INL to continue to work together to advance nuclear fuel predictive capabilities. In FY16 the TREAT M\&S team informally initiated the process to begin regular interactions and communications, to provide a unique foundation for fostering cohesive and productive interactions between experiments and models. The remainder of his report provides an overview of these activities, to demonstrate their need for mission connectivities and importance to the success of the TREAT mission. 


\section{Stakeholder Needs}

For the purposes of this discussion, the term stakeholders refers to the parties with near team interest to TREAT modeling and simulation: restart and operations, experiment design, instrumentation, modeling and simulation teams and of course the Department of Energy. On February 24, 2016 a kickoff meeting entitled "Merging Startup Testing and Modeling Development Efforts" was held to facilitate an open discussion of current and anticipated activities. A second meeting was held May 5, 2016 after the international reactor physics meeting PHYSOR 2016 in Sun Valley Idaho to take advantage of the availability of national and international experts related to transient reactor testing, "Transient Test Reactor Physics Workshop." Many participants were from INL, but the meeting included staff who support the CABRI transient test reactor at Cadarche, France, as well as other parties supporting INL research related to TREAT. On June 6, INL took the occasion of a visit from the NEAMS National Technical Director, Dr. Chris Stanek, to hold another stakeholder's meeting to brief Dr. Stanek on the status of project integration. The "TREAT-NEAMS Technical Meeting" was held for the purpose of discussion and planning to continue to ensure that the TREAT restart, operations, experimentation, and NEAMS modeling and simulation efforts were tightly coordinated. Finally, the NEAMS Integration Product Line lead, Dr. Brad Rearden, visited INL August 30 though September 1 to learn more about connections between each of the TREAT teams.

From these meetings, relationships between $M \& S$ and other activities have been identified. Typically these relationships are two-way. The following subsections provide an overview of the results of these discussions.

\subsection{Operational Needs}

Because the restart of TREAT cannot rely on availability of validated modeling tools for restarting the reactor, restart will be accomplished using historical methods for the prediction of reactor performance. It is well known that use the historical methods gives larger uncertainties than desired in the prediction of reactor performance and requires a significant number of trial runs to tune the reactor operating parameters to meet experiment goals. Once TREAT resumes operations, measurements can be taken to help validate new modeling and simulation methods. New methods, once validated with this data and put into production usage should be able to decrease the number of trial runs required to tune the reactor operating parameters for experiment needs. 
The historic method of operating TREAT required the use of flux wires to predict the power in the test fuel as a function of power in the reactor for the experiment configuration. The net power deposition was estimated by the use of a steady state power coupling factor (PCF) multiplied by the TCF described earlier. The power coupling factor is a ratio of the power generated in a sample in the experiment region to the core power itself. The numerator of the PCF ratio was determined from gamma activity - both a relative activity and an absolute fission activity. The relative activity measurement was performed with a $\mathrm{Ge}(\mathrm{Li})$ detector with a $5 \%$ estimated uncertainty. The samples used for absolute fission activity were taken as a sampling from the whole flux wire and they were dissolved and an aliquot was analyzed with a $\mathrm{Ge}(\mathrm{Li})$ detector looking for La-140. The estimated uncertainty was $2.5 \%$ for the absolute fission activity. On the other hand the PCF denominator comes from the neutron chambers or the chamber used to determine the core integral power.

Today, using flux wire runs to predict how an experiment would perform during reactor transient tests lacks the fidelity desired to truly understand the experiment conditions. With better modeling capabilities, detailed experiment conditions could be simulated and understood well in advance of the planned experiment, with flux calibration runs used to confirm the modeling results. With experiment conditions better understood, some of the more conservative assumptions used in safety analysis could be reduced, allowing margin for more experimental capability.

To this end, restart personnel have begun working with the other stakeholders, especially the modeling and simulation team. In May, restart staff participated in a full day BISON/MAMMOTH workshop. This provided a hands on demonstration of the status and capabilities of the codes at that time. Subsequently, the M\&S team assisted in installing MAMMOTH on restart staff computers, supplied access to TREAT core models and provided on-site support to help understand the input and how problems are run.

The restart team has also requested and received input from M\&S and benchmark development personnel on the types of startup physics testing desired to help validate transient physics codes. The physics testing interactions are expected to increase as the details of the test plans are refined in early FY17. These interactions, which will also include interactions with instrumentation experts, will continue to define not only the desired measurements but also the key locations to be instrumented and the number of different types of measurements that could be performed within the allotted schedule. 


\subsection{Experiment Design and Experimentalist's Needs}

Clearly, the first data of interest to begin an experiment design would include an accurate prediction of neutrons and gammas arriving at the target region as a function of time, energy, direction, pulse shape, etc. From that, an estimate of reaction rates within the target will be needed. To a first approximation this can be estimated through a neutronic-only solution; however, because of the nature of high power excursions, fuel samples will be also exposed to coupled mechanical, thermal and possibly hydraulic events in tandem with the power transient. Multi-physics modeling will be crucial in experiment simulation during a transient. While such modeling will be approximate in early simulations, it will be the results of experiments that were designed by simulations that will be used to inform the various physics models. This process will assist in the development of an embedded infrastructure for experimental design development in that environment.

Early simulations may also be able to provide specific guidance on the priority of future measurements and the required accuracy for such assessments, through sensitivity studies. Using advanced simulation tools, the investigator may focus on sensitivity studies to understand the mechanical response of a system to best design an experiment. MOOSE-based tools are under development to support global sensitivity studies, linking fluctuations and uncertainties in fundamental underlying data and models to those of engineering-scale observables, thus providing the quantitative foundation of a science-based, engineering driven research program.

As indicated earlier, results of future experiments will provide data to be able to improve the understanding of the complex physics of fuel power transients. In a very circular fashion, as computational models begin to come into alignment with observed interactions, the experiments will serve to validate the computational methods being used for experiment design. Figure 2 illustrates the circular relationship between these concepts.

The potential impact of MAMMOTH analysis for TREAT modeling and simulation has often been described as a way to reduce the number of calibration experiments required before approval and operation of a final transient. While this is true to some degree, historic calibration runs were performed predominantly with simple fission wires and reusable hardware and could be run as quickly as two per day. Hence the cost difference between running tens of calibration test versus just a few is essentially reduced to the man-hours required to perform said tests. Certainly the cost of staff time for a week of calibration tests would not be insignificant. However, the stronger value for NEAMS tools more likely lies in the ability to reduce time and money spent in experiment design iterations, some of 


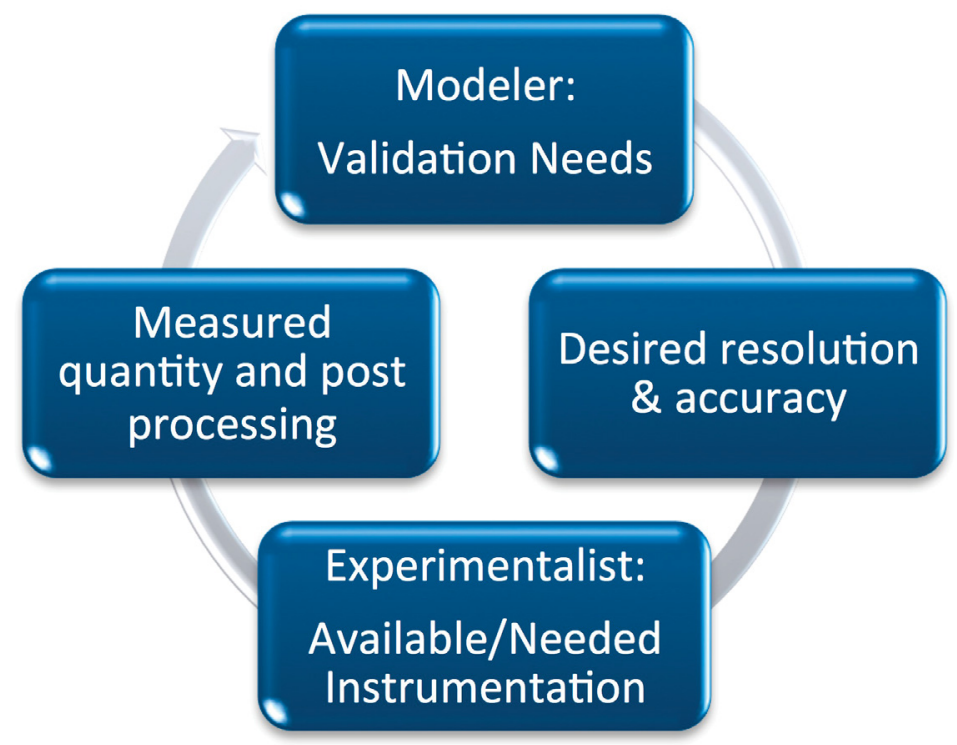

Figure 2: The ideal experiment is an evolving target based on iterative exchanges between experiments and modeling.

which will not occur until after the first calibration measurements are made. The potential for these iterations results largely from current tools and their inability to account for all of the salient time-dependent physics in TREAT experiments.

MAMMOTH physics analysts have already begun to support experiment design to predict the transient response of experiments loaded in a experiment vessel design. While Monte Carlo MCNP calculations are sufficient for understanding the neutronic interaction of a vessel design concept with the core at steady state, this approach is wholly inadequate in predicting the same interactions during a transient. The spectral shift in TREAT that ends the power excursion for a self-limiting transient has a significant effect on the experiment itself. Historical pre-test calibration measurements were used to characterize the change in the coupling of the experiment to the core over the length of a transient due to the neutron spectrum shifting as the transient progresses and the core temperature increases due to increasing power deposition; these measurements were used to determine a transient correction factor (TCF). For current designs, the core is not available to experimentally estimate TCFs and make necessary adjustments to the design concept. Hence, a MAMMOTH model of the experiment design is under development to provide the ability 
to obtain preliminary estimates of TCFs and to improve the physical understanding of the relationship of the experiment vessel and its contents with the core over the length of the transient. Section 4 provides a detailed explanation of the first vessel planned to support transient experiments and its needs from advanced modeling and simulation capabilities.

\subsection{Instrumentation Needs}

Coupled closely to experiment design and modeling efforts, the TREAT transient testing program has initiated a dedicated instrumentation program to support the objectives of near-term transient testing in static environments (with pellet-clad interaction physics being a primary target), forced convection environments, and beyond, to next generation testing purposes. The ultimate goal of the transient testing instrumentation program is to reduce the uncertainties of the experimental conditions (including neutron flux, heating, dose/fluence, etc.), while simultaneously monitoring physical behavior of interest (including, for example, temperature, dimension change, relocation, microstructural evolution, etc.). The transient aspect of these measurements is particularly challenging in some cases with time scales ranging from milliseconds to minutes and length scales ranging from microns to meters. The close association of the instrument program to experiment design and analysis efforts provides model-informed instrumentation targets for experiment phenomena of interest. This is a classical illustration of the circular nature of the modeling and experimentation process shown earlier in Fig. 2.

In addition to having a critical role in instrument-needs identification, modeling is already playing an increasing role in developing and qualifying instrumentation for implementation in TREAT experiments. Good examples of current and ongoing efforts to use modeling to support experiment instrumentation include both the micro-pocket fission detector (MPFD) and the boiling detector under development for use in the current Static Environment Rodlet Transient Test Apparatuses (SERTTA) design (see Fig. 3 and SERTTA design specifics in Section 4). In each case, modeling plays a key role in design optimization in terms of sensor placement, geometry, and materials. Additionally, modeling efforts are focused on developing methodologies for predicting sensor output based on targeted measurement parameters. In the case of TREAT, advanced modeling tools could provide needed spatial and temporal information regarding radiation fields and evolving thermomechanical phenomena. For example, the optimal design of fissile layer thickness and materials in MPFD could be better facilitated with time and spectral-resolved prediction of radiation fields in the experiment [20]. Beyond instrument implementation needs, a long-term goal for advanced

modeling of experiments could include simultaneous simulation of an instrument alongside 
the test specimen, where the instrument response to the measured parameter results from the true multi-physics and multidimensional nature of the experiment.

More details on MPFD design are provided in Sect. 5.
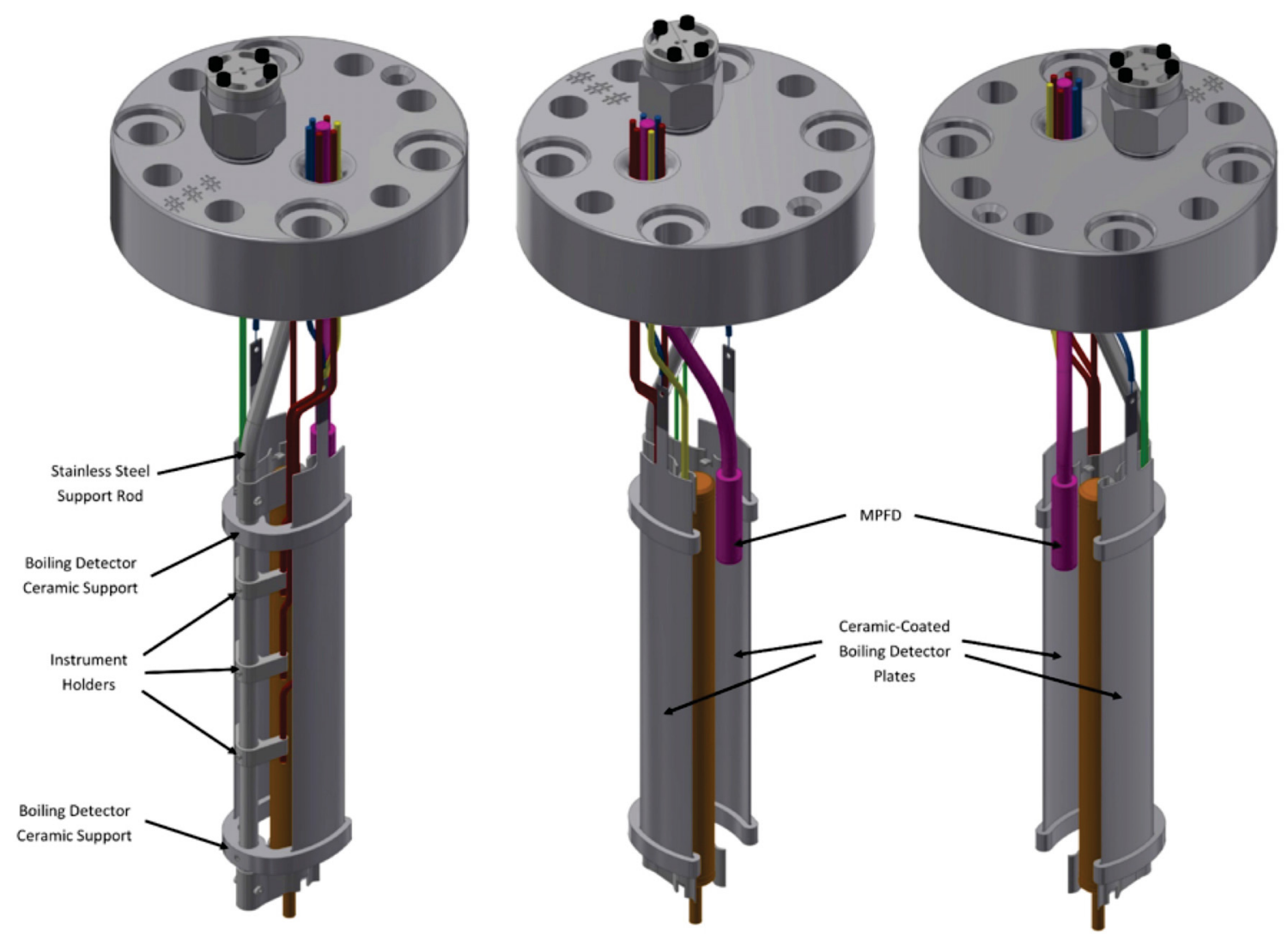

Figure 3: ATF instrument array within a SERTTA capsule.

\subsection{Benchmark Development Needs}

Analysis of historic TREAT transient data has been ongoing under current DOE NEUP and IRP projects to gather and evaluate data that could potential serve as benchmark data for validation of modern simulation and modeling efforts. As a minimum, these efforts would demonstrate the lack of detail recorded and areas of significant bias and uncertainty that would impact the development of benchmark models. Lessons learned from these 
efforts can effectively feed into future physics testing and experiment design in TREAT for the preparation and measurement of modern experimental data sufficient to validate our modern nuclear data and toolsets.

The extra time and effort taken to evaluate and prepare benchmark models and measurements, such as those encountered in the international Handbook of Evaluated Reactor Physics Benchmark Experiments [21] provide high-quality, extensively peer-reviewed, data utilized to validate current and future neutronics codes and nuclear data libraries. Such practice applied to TREAT restart physics tests and experiments would greatly enhance their usability in supporting current and future NEAMS activities.

An integral byproduct of benchmark evaluation of historic and upcoming TREAT measurement data would further provide the means to not only effectively evaluate the capabilities, limitations, and unknowns of the current HEU-fueled TREAT core, but provide insight into the capabilities and needs for a converted LEU-fueled TREAT core design. TREAT LEU conversion should adequately address, and hopefully enhance, not only current HEU core capabilities, but also provide a more well-known material property environment that would better enable modeling and simulation of the TREAT core, not just for neutronics evaluation, but multiphysics effects during transient experimentation.

\subsection{MAMMOTH Modeling and Simulations Needs}

As indicated earlier, current experiment designs are being evaluated in lieu of in-core TREAT measurement using conventional methods, tools, and data, supplemented by unvalidated transient calculations using MAMMOTH. To be able to quantify the accuracy of MAMMOTH, qualify it for safety analysis and to provide confidence in its simulation capability, validation of MAMMOTH calculations is imperative. Instrumentation strategically designed and placed throughout TREAT during restart physics testing and experiments during preliminary transient testing will provide measurements necessary to validate current and future modeling and simulation capabilities.

Following initial critical measurements and startup testing, currently scheduled for late 2017 and early 2018, a phased approach is planned for reestablishing a detailed understanding of TREAT operations and physics. These phases are described in the following subsections. 


\subsubsection{Reproduction of the M8 Calibration Tests}

The first phase will be based on the M8 Calibration series of measurements performed in 1992 and 1994 [15]. This test series will reconfirm similar measurements completed before the TREAT reactor was placed in standby using the available M8 calibration vehicle. The results will demonstrate that the TREAT reactor is irradiating samples in such a manner as to be able to reproduce historical experiments. This campaign will require approximately one month of reactor time and will require the following:

- M8 calibration vehicle

- Loop Handling Cask

- Flux and fission wires

- Flux/fission wire counting equipment

- Access to and perhaps priority for the MFC radiochemistry lab

Although this work is being performed to demonstrate that TREAT is being operated in the same manner as historical operation, the data will be better qualified with direct access to raw data. It is hoped that flux and fission measurements will be performed in numerous spatial locations, beyond what was done in the original M8CAL series, to provide more information on spatial resolution. However, given the intent of this measurement series, such supplementary measurements my not come to fruition.

\subsubsection{Multi-SERTTA Calibration}

This test will be used to confirm Multi-SERTTA modeling and engineering design work. It will verify the axial flux profile in the vehicle and will be used to tune the flux and energy deposition profiles for the four locations in the Multi-SERTTA vehicle. This will require approximately two months of reactor time and, in addition to the Multi-SERTTA vehicle, all of the hardware and access requirements listed in Sect. 3.5.1 will be required here. The M8 calibrarion vehicle will be used for initial measurements of transient correction factor as a function of axial position. These measurements will be the first confirmation of computed TCF from earlier simulations, and to fine tune the Multi-SERTTA vehicle. 
It is not clear if MPFDs will be available for testing at this time - however, this would also provide an opportunity to determine if MPFD design calculations match reality. The use of MPFDs in the Multi-SERTTA design is discussed further in 4.

\subsubsection{TREAT Temperature Measurements}

This phase will be the first set of measurements requested to begin data collection specifically for validation of modeling and simulation capabilities. This phase is intended to support measurement of temperatures in the core, to develop a three-dimensional (3D) temperature profile and support computer model validation. In addition, during development of the temperature profile, the negative temperature coefficient will be confirmed and further refined to improve kinetic modeling capabilities. This work will require approximately two months; the form of the measurement equipment remains to be determined. Temperature measurements by traditional means will be limited to the few existing thermocouples or use of new thermocouples located in air channels adjacent to fuel element cladding. Clad temperatures will not be representative of fuel temperatures unless the core is held at a constant power level for until equilibrium is reached. Other options, requiring some development, would be (1) thermocouples added to a refurbished element, that could be moved around the core, or (2) a thermocouple installed within a small fuel sample that would respond to local fluxes in the same manner as adjacent elements. The locations (axially and radially) would need to be predetermined, along with desired peak or average temperature targets. This work would require one to two months reactor time.

\subsubsection{TREAT Flux Measurements}

This phase will seek to measure the neutron flux throughout the TREAT core and develop a 3D flux map. Again, this information will be used for code validation purposes. This work will require approximately two months of reactor time (perhaps in parallel with other measurements), and will again require flux and fission wires and access to counting equipment and radiochemistry facilities. Placement of wires would be easier than for temperature measurements, and fluxes within air gaps would be representative of the four surrounding elements. Because of the relative ease with which such measurements could be performed and the simplicity of the instrumentation requirements, this work could potentially be extended to a significant number of locations radially and axially. The availability of MPFDs could potentially accelerate the measurement process by providing real time data. 


\subsubsection{Characterize Transient Capabilities in TREAT}

In support of reactor operations needs, this phase is planned to perform partial and full transients to demonstrate the capability of the TREAT Automatic Reactor Control System (ARCS) to control a variety of transient shapes. This work would demonstrate the ability of TREAT reactor engineers to generate transient prescriptions for defined transient shapes. Additionally, this work would further improve understanding TREATs capability to deposit energy during a transient and ultimately be used for validation of kinetics calculations.

\subsubsection{Neutron Lifetime and Beta Measurements}

This phase will focus on the measurement of the neutron lifetime and beta associated with the TREAT core. While these have been measured previously, advances in instrumentation and detection capabilties should allow more precise measurements. This work would provide data that would improve kinetics modeling. This will require approximately one month and would require a specially-fabricated oscillating rod installed in the experiment location and installation of the reactivity measurement system that the M\&S team is currently developing. This system is described in more detail in Sect. 6.

\subsubsection{Neutron Spectrum Characterization}

Experiments will be necessary to characterize the neutron spectrum within specific locations of the TREAT core during a range of operating conditions (e.g., temperature, lowerpower transients) to support validation of analysis methods. Selected foils of predetermined materials would be needed, and a spectral unfolding process similar to that described in Ref. [22] would be employed for spectrum measurements. Because the foils must be taken to a counting facility post irradiation then decayed to background levels before being used again, either a number of sets of foils will need to be procured, or measurements limited selected irradiations. These measurements must be performed at a number of fixed temperatures to be able to capture the effect of temperature on spectrum. This will be a key measurement to validate the calculation of spectra during high-power transients.

INL staff are currently providing direct support to other stakeholders for near-term needs, both in terms of MAMMOTH-based simulations as well as other technologies. Section 6 provides a summary of this work. 


\section{Advanced Modeling for the Multi-SERTTA Irradiation Vehicle}

TREAT static-environment experiment vehicles are being developed to enable transient testing of Pressurized Water Reactor (PWR) type fuel specimens, including fuel concepts with enhanced accident tolerance (Accident Tolerant Fuels, ATF), as well as other types of compatible specimens. These types of experiment vehicles have been termed Static Environment Rodlet Transient Test Apparatus (SERTTA).

The first of these SERTTA devices has been designed in detail by the INL transient experiment team and is currently undergoing final prototype testing and design qualification for use in TREAT. This design can accommodate four rodlets, each within its own hermetic boundary, and is known as the Multi-SERTTA. The Multi-SERTTA irradiation vehicle provides support for specimens and instrumentation, affords desired boundary conditions, and safely contains the hazards associated with transient testing of nuclear fuel. A larger single-vessel design, known as the Super-SERTTA, will be deployed shortly thereafter for enhanced data collection capabilities on pre-irradiated fuels. Additionally, flowing loop designs for water and sodium-cooled fuel systems will follow. See Figs. 4 and 5 for illustrations of the current Multi-SERTTA design [23]. The design of instrumentation in the Multi-SERTTA design was illustrated earlier in Fig. 3.

The Multi-SERTTA design has already revealed several challenges/opportunities for advanced modeling and simulation. It is also reasonable to expect that other future design efforts (e.g. water loop) will follow the same trend. The design effort is currently described into two phases.

\subsection{Phase 1}

Phase 1 efforts are planned to be the first transient tests of nuclear fuels in the restarted TREAT under the ATF-3-1 transient test series. Phase 1 will address the use of MultiSERTTAs with fresh fuel rodlets starting from PWR conditions. Test objectives mandate precise energy injection targets to demonstrate that ATF fuel designs can withstand current regulatory limits. Each of the four SERTTA vessels will be shrouded to varying degrees with neutron-absorbing flux collars to tailor the reactor's axial flux profile and enforce uniform specimen PCFs in each specimen. Leveling the PCFs across the cores axial profile in this manner has not been attempted historically in TREAT, but is necessary for these 

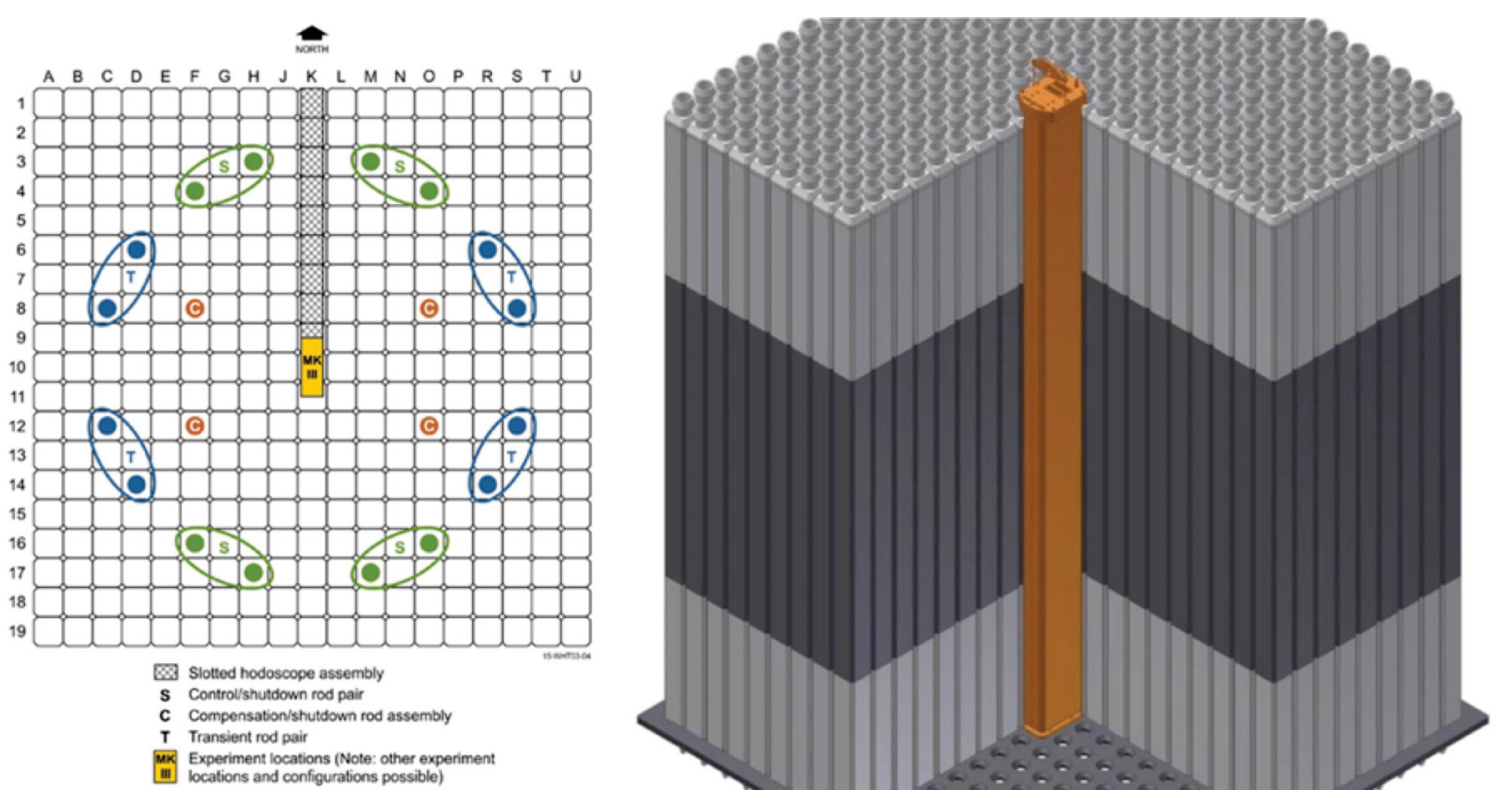

Figure 4: Example core map (left), package type vehicle in TREAT core section view (right).

experiments to support the specimen throughput needed for ATF testing. Accomplishing this design objective necessitates an understanding of TREAT axial flux profile and how it evolves throughout a transient.

The planned Phase 1 transient will be akin to a PWR hot-zero power reactivity initiated accident (HZP-RIA) as it will start from near-zero power with a single step reactivity insertion and terminated moments later with reinsertion of the transient rods to minimize pulse width and control core energy release. The total duration of this transient will be on the order of a couple hundred milliseconds while releasing several hundred MJ of core energy. This type of transient represents some the most-rapidly changing nuclear conditions that TREAT can provide. The core energy release is currently being predicted by point kinetics using historic temperature feedback tables. The core-to-specimen PCF values are predicted during steady state solutions using the MCNP5 Monte Carlo code [8]. Point kinetics predictions can provide reasonable estimates of the cores average behavior with respect to time (insofar as the feedback tables are accurate), but lack the ability to predict 3D spectral or spatial shifts. Estimates of 3D behaviors are particularly important for the Multi-SERTTAs intricate design, since none of its geometries can be adequately represented by simplified models (e.g. axisymmetric, two-dimensional approximations, etc.). As such, Monte Carlo 


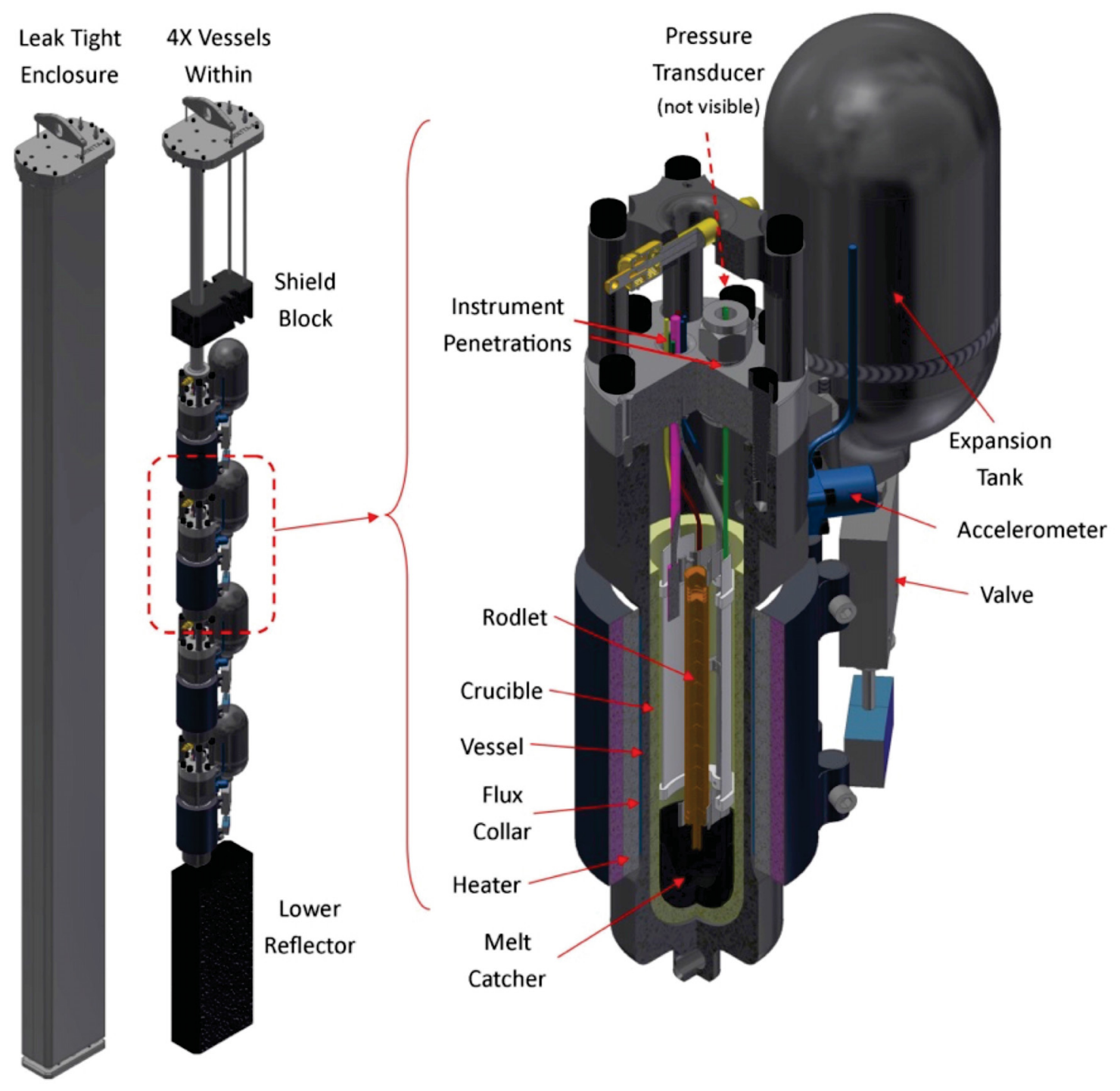

Figure 5: Multi-SERTTA Full Assembly

methods are used to predict 3D steady state behavior, but lack the ability for full coupling to implicitly predict time-dependent effects.

For these reasons, Multi-SERTTA must also be accompanied by an equally intricate calibration (-CAL) experiment vehicle. Multi-SERTTA-CAL will permit empirical measure- 
ment of PCF values and TCFs with radiochemical dosimeters. The Multi-SERTTA-CAL vehicle will also include real time fission detectors capable of providing both fast and thermal neutron flux. The Phase 1 Multi-SERTTA-CAL plan includes two complete iterations of these measurements, requiring several months of schedule, before final transient tests can be performed using Multi-SERTTA. The primary goal of these calibration vehicle iterations is to fine tune the flux collars to achieve specimen energy targets in the final transient. If more than two iterations are necessary, it will fall outside of the ATF-3 planning basis. As such, any information from advanced modeling and simulation that can help indicate the spectral, spatial, temporal, and 3D behavior of the Multi-SERTTA could be crucial in facilitating success. The status of such calculations, already showing 3D effects, is discussed later in Sect. 6.3.

To understand the scenario more completely, it is first important to understand how TREAT will operate Phase 1 Multi-SERTTA transients. Figure 6 illustrates three rod position cases as currently evaluated by MCNP models. Case 1 (left) represents the startup case with transient and compensation rods fully withdrawn, and where criticality is achieved by withdrawing control/shutdown rods. Case 2 (center) represents the case just prior to transient initiation where the transient rods are reintroduced into the core, swapping them with control/shutdown rods so that a prescribed reactivity step is available for insertion (k), and the core is operating at very low power, approximately 50W. Finally, case 3 (right) represents the rod positions during the transient after they have been rapidly withdrawn from the core. The Multi-SERTTA flux collar design is currently based on counteracting the steady state solution for the case 3 axial flux profile. This assumption is thought to be fairly reasonable because the transient initiates from very low power, the transient rods move very quickly, and the reactor period will be on the order of tens of milliseconds. As a result, the transient rod should be entirely withdrawn before the core reaches appreciable power levels.

The transient will be terminated, or clipped, by rapid reinsertion of the transient rods coming in from the top of the core. It is hypothesized that the effective local transient experienced by each vessel unit within Multi-SERTTA will be successively clipped as the rods proceed downward, at least until the core becomes subcritical again. No attempt has yet been made (or really is possible to do) to model this effect with current methods. This complication, along with complex spatial/spectral interactions between the water within Multi-SERTTA, delayed neutrons, etc. all strongly suggest that the initial flux collar design (based entirely on a steady state solution for case 3 rod positions), will be found somewhat off-target during the initial Multi-SERTTA-CAL measurements. Predictive tools with the ability to inform the designer to the effects of these complicated phenomena can help design the Phase 1 Multi-SERTTA flux collars. Additionally, these tools can be used to describe 


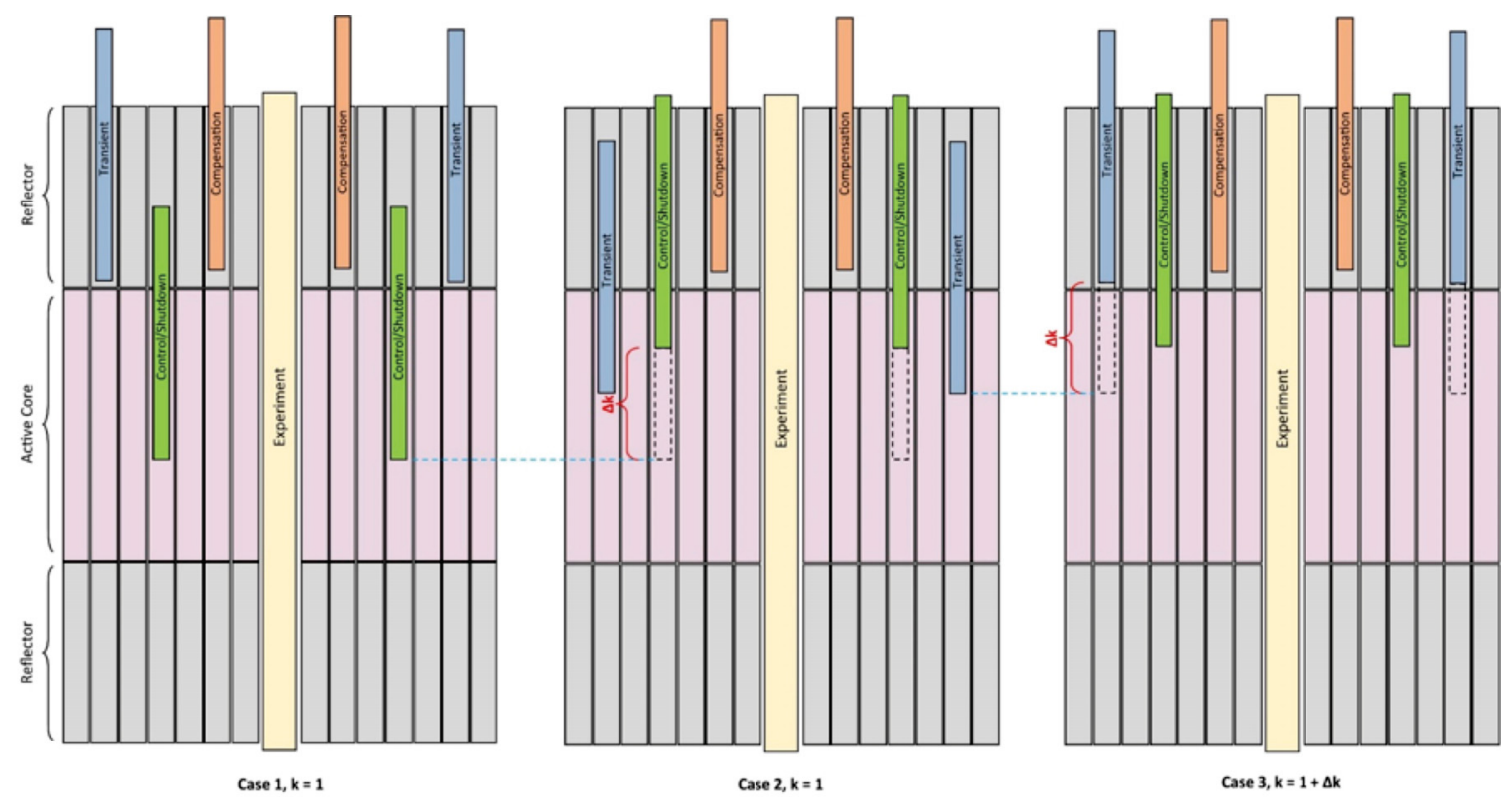

Figure 6: Rod Position Cases

conditions for transient experiments post-test based on as-run reactor power, rod positions, etc. Along these lines, MAMMOTH-based foundational work has already been initiated to describe the axial dependence of PCF and TCF values in Phase 1-type transients.

MCNP models have shown that the inclusion of light water in the Multi-SERTTA has the effect of increasing the PCF due to water moderation [24]. Studies have also shown that this effect depends both on spectrum and backscatter of fission-born neutrons emitted from the specimen. MAMMOTH could be used to account for rapid spectral shift and delayed neutron effects to provide more accurate predictions of PCFs and TCFs. Conveniently, the Phase 1 transient does not need to be overly concerned with moderator void effects on power coupling (i.e. water boiling within Multi-SERTTA), due to the brief nature of the pulse and the relatively long amount of time it takes for heat transport through the cladding and to the coolant, such that the nuclear event and the thermal-hydraulic event are mostly separated in time. This simplification, if true for Phase 1 transients, will almost certainly not be valid for some more-complexly shaped transients to be attemped in Phase 2 . 


\subsection{Phase 2}

Phase 2 is somewhat open ended, but will at a minimum include extension of the MultiSERTTA design envelope to permit testing of ATF fuel and cladding concepts $\left(\mathrm{U}_{3} \mathrm{Si}_{2}\right.$, FeCrAl, SiC, etc.). Phase 2 may also address the general extension of the Multi-SERTTA useable envelope to include pre-irradiated or plutonium-bearing specimens, different flux shaping collar schemes, a wider range of starting water conditions, and different test environments such as inert gas, steam, and liquid sodium. Phase 2 will also include deployment of the Super-SERTTA vehicle and flowing water and sodium loops.

Modeling with the NEAMS multi-physics application MAMMOTH is anticipated to present a sizeable benefit in better prediction of PCFs and TCFs for more rapid experiment design in Phase 2 with the potential for performing only one calibration iteration. Experiments including pre-irradiated specimens or specimens with significant plutonium content will be challenging to implement in representative dosimeters in calibration measurements. Since the spectral interplay between coolant, TREAT's rapid spectral shift, and the presence of other fissile isotopes in these specimens is likely to be complex, this NEAMS tool could potentially provide a higher fidelity correction factor between these types of specimens and the measurements made on fresh calibration dosimeter specimens. From a historical perspective of TREAT experiments, the complicated nature of predicting power coupling for pre-irradiated fuel is described for the M-series tests, in Section IV.B. of reference [25]. These experiments were performed late in the TREAT program, when it was quite mature, prior to the suspension of TREAT operations in 1994. This example sheds some light on the opportunity for advanced modeling tools to accelerate and improve the process.

Phase 2 transients will also include transient shaping and/or variable thermal-hydraulic conditions. A simple example would be power ramps (slower than RIA) up to various boiling conditions. A more elaborate example would include prototypic Loss of Coolant Accident (LOCA) simulations where the TREAT transient is shaped to transition from PWR-prototypic powers to decay heat simultaneous to the intentional depressurization of the vehicle. These types of tests are all planned for future experiment series. One of the greatest benefits that MAMMOTH-based modeling of TREAT experiments can realize is to develop the capability to predict these coupled phenomena, especially in water environment tests. Water moderator density changes from boiling, and resulting changes to PCF, simultaneous to the nuclear aspect of the transient, must be coupled for accurate experiment design and post-test analysis. Envisioning a calibration vehicle design that physically simulates such a scenario for empirical measurement would be extraordinarily challenging. MAMMOTH will be needed to couple experiment thermal-hydraulics to transient 
core physics, accurately assess transient fission heating in the specimen, and help TREAT realize its full experimental capability.

As part of Phase 2 and beyond, TREAT experimental opportunities are quickly evolving and becoming more varied with complex scientific missions. Studies of fuel behavior under a full range of accident conditions and scenarios requires capability to model coupled multi-physics phenomena involving classical thermal, mechanical, and chemical effects. The addition of nuclear effects makes the integral problem significantly more complex. In addition to power coupling effects from reactor rod positions, pre-irradiated fuel microstructure, and evolving moderator effects, historical and future experimental needs require the capability to model fuel movement evolution and its effect on specimen power that will be captured by the hodoscope and other possible instrumentation. Historical tests used a variety of approaches to handle such events for example relying on adjustments determined from previous experiments and/or thermal measurements in surrounding heat sinks [26]. These effects of interest include within-pin fuel movement due to thermal expansion, fuel melting, and/or fission gas release. In some cases, cladding breach allows fuel to be dispersed into the coolant where it may be swept from the core or dispersed in a capsule, frequently involving rapid phase change of the coolant. In all cases, power coupling effects between the reactor and the test specimen play a crucial role in understanding experiment behavior. 


\section{Design and Modeling of Micro-Pocket Fission Detectors}

Micro-Pocket Fission Detectors (MPFDs) are under development for in-core neutron flux measurements at TREAT and other experiments at INL. The sensitivity of MPFDs to the energy dependent neutron flux at TREAT has been determined for $300-\AA$ thick active material coatings of ${ }^{242} \mathrm{Pu},{ }^{232} \mathrm{Th}$, natural uranium, and $93 \%$ enriched ${ }^{235} \mathrm{U}$. Electrode thickness and active material thicknesses for the expected range of values was determined to have negligible effects on the neutron flux in the immediate vicinity of the experiment. Selfshielding effects in the active material of the MPFD was also confirmed to be negligible. Finally, fission fragment energy deposition was found to be in conformance with previously reported results.

Experiments are already being designed to utilize the TREAT facility following the restart. A determination of the neutron flux during transient experiments is necessary to properly characterize nuclear fuel and materials for advanced reactor designs. Self-Powered Neutron Detectors (SPNDs) have been previously deployed for the high thermal neutron flux (up to $10^{17} \mathrm{n} \cdot \mathrm{cm}^{-2} \cdot \mathrm{s}^{-1}$ ) during transient experiments at TREAT [27], however alternative methods for neutron detection using an advanced fission chamber design are under investigation.

MPFDs have been constructed and tested at other nuclear research facilities in the past, successfully tracking neutron flux as high as $10^{15} \mathrm{n} \cdot \mathrm{cm}^{-2} \cdot \mathrm{s}^{-1}$ during power transients [28]. Continued development and validation of MPFDs at nuclear reactor test facilities (including TREAT) is an important enhancement of advanced reactor testing [29]. Gamma-ray discrimination, small size, and minimal neutron field perturbation are the primary advantages of MPFDs [30]. In contrast to most other in-core instruments designed for highneutron-flux operation, MPFDs can operate in pulse-mode, allowing for the contribution from non-neutron-induced events to be easily discriminated [30]. Their small size also enables MPFDs to be assembled into arrays with multiple detectors in close proximity. This feature is of particular interest for a transient testing experimental design for the MultiSERTTA [23]. The Multi-SERTTA design was described in detail in Section 4.

Testing of experimental fuel in accident conditions is an important step in qualifying new reactor fuels and determining the safe operating conditions for advanced reactor designs [31]. MPFDs will be deployed along with other advanced instrumentation in the MultiSERTTA vehicle in the central test channel in TREAT as shown in Figure 7.

Dimensions for the MPFD array were determined based on estimates from the evolving Multi-SERTTA design, and are illustrated in Figure 8. The MPFD design consists of neu- 


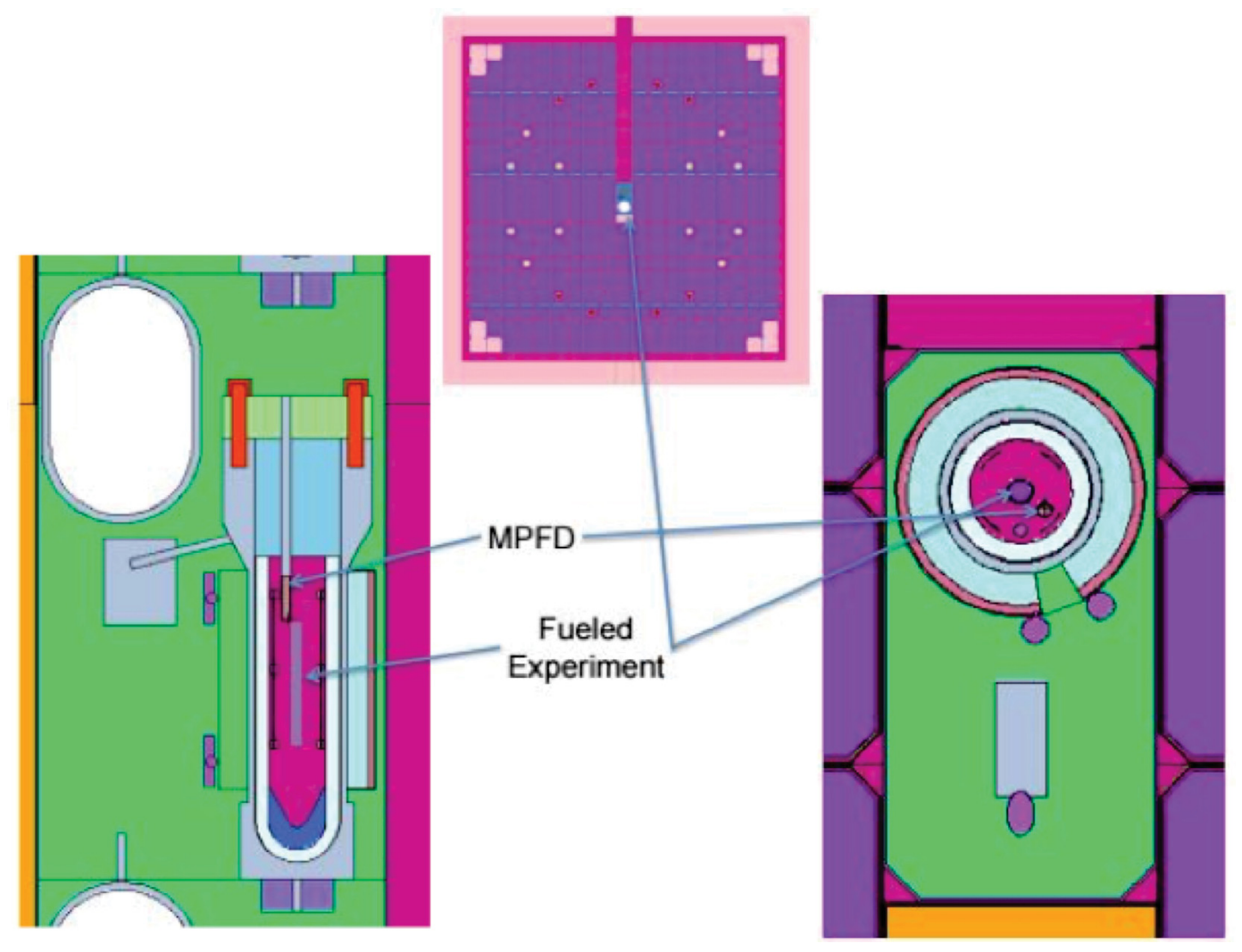

Figure 7: Multi-SERTTA and included MPFD assembly will be deployed within the central test channel at TREAT.

tron sensitive material which is deposited onto thin Ti/Pt substrates within the sensor gas volume. Along both sides of the gas chamber run parallel electrodes which are used to measure the ionization within the gas chamber from fission fragments [30]. The bulk of the MPFD is constructed from insulating alumina $\left(\mathrm{Al}_{2} \mathrm{O}_{3}\right)$, which is chosen for its radiation hardness and high-temperature stability. The MPFDs are stacked, with the electrode wires for each detector off-set from one-another by $45^{\circ}$ to allow readout of 4 sensors simultaneously. The MPFD array is encapsulated within an Inconel-625 sheath, and connected to custom-made extension cable of similar materials. The MPFD model was developed in MCNP and inserted within the Multi-SERTTA model shown in Figure 8. However, these remain static evaluations - detailed calculations performed using MAMMOTH will be required to fully characterize transient MPFD performance until actual tests can be performed in the reactor. 


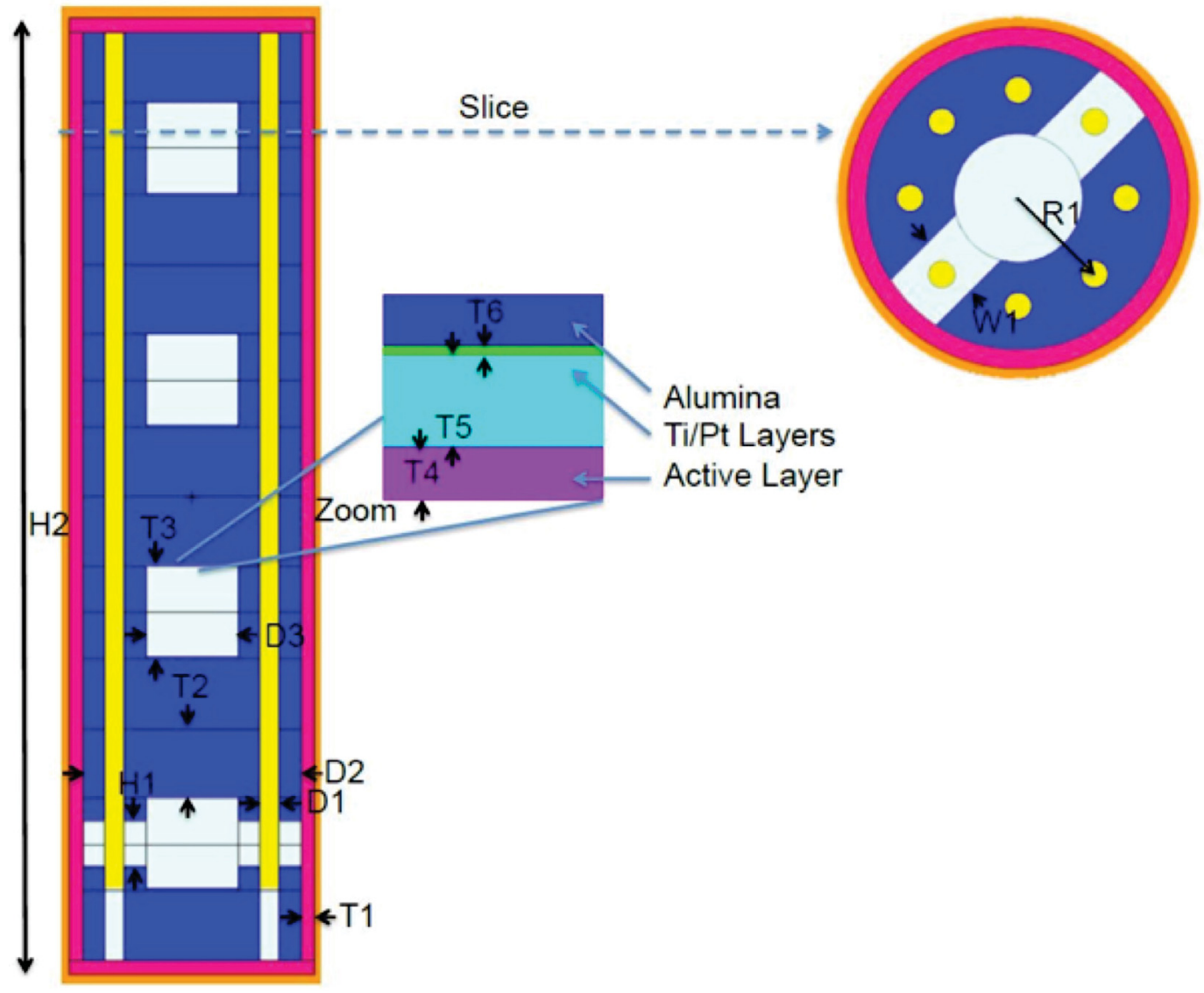

Figure 8: Illustration of the most current micro-pocket fission detector design 


\section{Modeling and Simulation Support}

Previous sections have highlighted general areas of collaboration between TREAT M\&S staff and other stakeholders vested in TREAT operation and restart, looking toward the long-term mission of M\&S support. However, the $M \& S$ staff is directly supporting current activities as well, under work funded by other organizations, building on team capabilities. This section describes some of the more significant activities initiated in FY16. While funded separately, each of these tasks have direct ramifications and interactions with development and refinement of MAMMOTH capabilities.

\subsection{Reactivity Computer}

One of the more visibile contributions to TREAT restart activities at the present time is in the development of software for a reactivity computer. This reactivity computer will shorten the time required to validate that the TREAT core is operating as expected following core alterations, and is anticipated to be useful for other core physics measurements. M\&S staff are combining practical experience and physics modeling skills to rapidly develop the software to support current reactivity measurements needs; this will provide a capability that has not previously been available for TREAT operations. In terms of operational applications, the reactivity computer will be used as the primary means of of monitoring and recording reactivity data as a function of time during operation of TREAT. The process of identifying the functional requirements of the reactivity computer has required close coordination with the TREAT engineering and operations staff to satisfy each of their specific needs, while the activities associated with building the reactivity computer has provided additional opportunities for collaboration with the TREAT restart and operations teams and has allowed for a better understanding of the processes by which data has been collected at TREAT in the past and the limitations to those data.

The reactivity computer will be a user interface that records the signals from the neutron detectors, and based on the signal history, determines the reactivity in real time. The program provides a graphical user interface which will record the raw data from fission chambers, and after signal processing, display the desired results. The computer will record various sensors in addition to the neutron detectors. These will include the control rod positions, thermocouple temperatures and the blower system information. At present, the reactivitiy computer is simulator that provides the ability to virtually adjust control rod positions; a point-kinetics solver simulates the core response following rod motion. Figure 9 shows the 


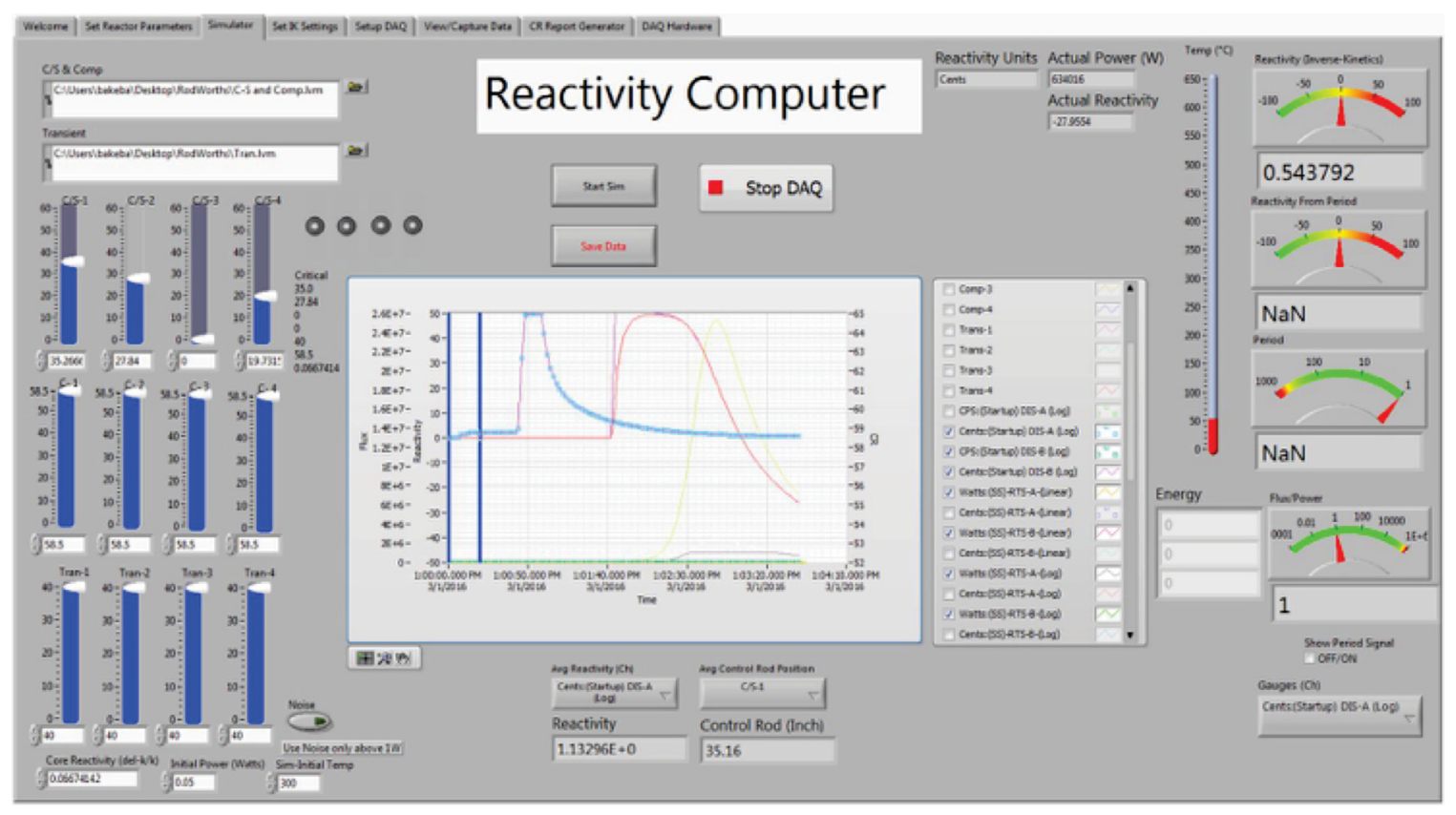

Figure 9: Reactivity Computer Simulator Layout

current reactivity computer interface. When deployed it will continue to provide a simulation mode for pre-transient simulations; however, the system will be directly connected to reactor data acquisition and rod position indicators to be able to provide real-time core behavior data. Thus, this represents not just a simulation effort but also a new form of operations software to directly complement operations activities.

\subsection{Flux and Power Estimations}

Instrumentation design for next generation TREAT instruments will require information on the flux as a function of space and energy during hypothetical transients. Such data will be readily available once M8CAL transient calculations have been completed, albeit based on calculations with incomplete validation. Work documented in Ref. [32] describes the state of work completed near the end of FY16 for steady state (pre-transient) calculations, with good agreement with a reference Monte Carlo solution and with historical measurements. Since that time, transient simulations have been initiated, with reasonably good agreement to measured data, although work remains to be completed. Once this has been completed, in 
early FY17, time dependent flux, fluence and reaction rates will be available for preliminary design. At this time, instrument designers are seeking a "ball park" estimate so that it is possible to scope out the feasibility of use of various detectors to interrogate an experiment as it is being pulled out of the reactor after a transient. Instrument design has also requested an estimate of average flux per power level for TREAT at steady state. Instrument design has also requested an estimate of average flux per power level for TREAT at steady state, which has been provided and verified with historical data. The calculation estimated a thermal flux of close to $8 \times 10^{8} \mathrm{n} \cdot \mathrm{cm}^{-2} \cdot \mathrm{s}^{-1} / \mathrm{kW}$. This ratio seemed very low, considering that typical power reactors are nearly an order of magnitude larger, at around $1 \times 10^{10} / \mathrm{kW}$. $\mathrm{n} \cdot \mathrm{cm}^{-2} \cdot \mathrm{s}^{-1} / \mathrm{kW}$. However, the calculated value was confirmed with a report that indicated that the thermal flux for TREAT is approximately $1 \times 10^{9} \mathrm{n} \cdot \mathrm{cm}^{-2} \cdot \mathrm{s}^{-1} / \mathrm{kW}$.

In support of restart activities, the $M \& S$ team was requested from the TREAT Integration Program to estimate the rod worth for each single control rod to ensure that no single rod removal could result in an accidental criticality. This was only a confirmatory calculation historical data showed that such an accidental criticality could not occur with the removal of any single rod, with an extremely large margin to account for uncertainty. Because these independent calculations confirmed historical data, control rods were able to be removed and inspected one at a time before beginning to test control rod drives.

These calculations were followed by a request to calculate assembly averaged axial power distribution for the TREAT core for a given core power level. This information was used to support core detector design and sensitivity analysis. At present, there are two potential problems in placement of modern detectors, (1) the spectral shift during a pulse affecting the response of the boron-based neutron detectors (which are most sensitive to thermal fluxes and will become less sensitive with spectral hardening), and (2) the high radiation immediately outside the reactor at the transient detector penetrations. The transient detectors are partially covered with cadmium to reduce their sensitivity to the energy distribution shift during a pulse. This is know to work reasonably well but is not perfect. It is unknown how many neutrons make it through the cadmium and interact with the detector, nor is it known if the flux above the cadmium cutoff is affected by the spectral shift. Calculations that will be done in the future by the $M \& S$ team will provide a post-test correction to neutron detector data. 


\subsection{Multi-SERTTA Modeling with MAMMOTH}

By far the most significant work performed in TREAT simulation has been evaluation of the Multi-SERTTA experiment vessel design. The Multi-SERTTA experiment adds a level of complexity that is unprecedented in TREAT modeling. The level of resolution has to be increased and optimized in order to obtain a viable simulation that produces meaningful results. The MAMMOTH modeling process has entailed three fundamental tasks:

- Preparation of appropriately weighted cross sections;

- Development of an analysis mesh that would capture the necessary details of the vessel design; and

- Assembly of the MAMMOTH input with appropriate control parameters and output specifications.

The preparation of cross sections, which is the most difficult step, is discussed in Section 6.3.1. The improvements in mesh generation are presented in Section 6.3.2. The description of the MAMMOTH input for this analysis is omitted, since the model does not include a large number of physics that require further explanation. Section 6.3.3 includes a discussion of the results from the initial steady state calculation of a small domain of the TREAT core with a SERTTA unit.

\subsubsection{Cross Section Preparation}

The Serpent [33] Monte Carlo code is used in the preparation of cross sections that are spatially homogenized over various material regions and energy condensed to 11 energy groups. One of the major challenges in this task is the determination of the constant (or flat) cross section regions that will be used in the MAMMOTH deterministic solution. Serpent identifies constant cross section regions based on the universe numbers provided in input. Hence the full Serpent model must be developed in such a way as to provide a direct correspondence between Serpent universes and the various constant cross section regions in the MAMMOTH model. This greatly complicates the geometric definition for the Monte Carlo input, which generally just follows the physical features of the geometry.

Several geometric features are simplified in order to expedite the modeling. Those include: 
- Approximation of the vehicle walls with a rectangular shape;

- Approximation of the expansion tank with a rectangular cylinder;

- Omission of the MPFD;

- Homogenization of the fuel rodlet support and horse shoes into a rectangular cylinder with equivalent mass and enough volume to conserve the water in the pressure vessel;

- Omission of additional small metal clips, screws, light pipes, thermocouples, wires, etc.

The cross section regions defined in each SERTTA unit include various concentric rings to model the pin, clad, water, pressure vessel internals as well as external heater and neutron filters. Views of the simplified Serpent model are shown in Figure 10. The pin is further divided into two radial regions, which increases the resolution near the surface of the pin. Two azimuthal division were also added to the pin and moderator in order to capture the expected flux asymmetry introduced by the slotted elements facing the experiment.

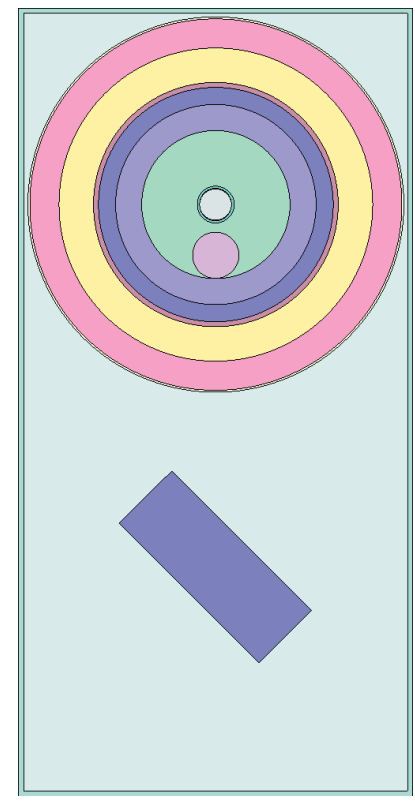

(a) $\mathrm{XY}$ view

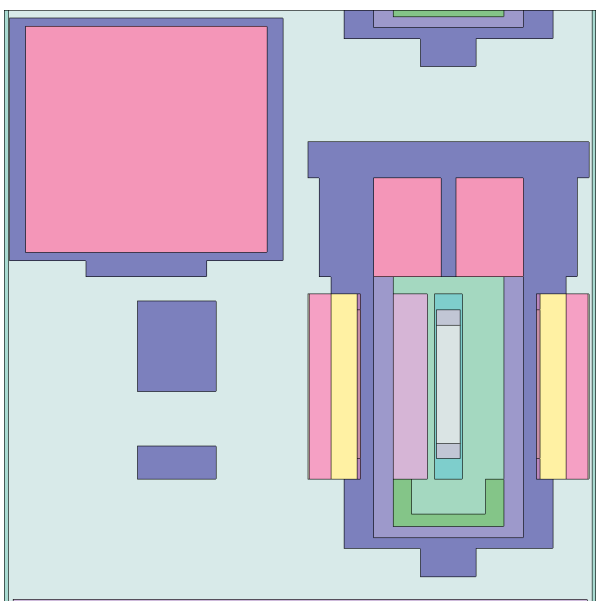

(b) YZ view

Figure 10: Serpent Model of the SERTTA Unit 
Two tests were conducted on simple configurations before proceeding to the full core: (1) an isolated single SERTTA unit and (2) a $5 \times 5$ supercell with a single SERTTA unit. The $5 \times 5$ supercell is depicted in Figure 11. The supercell consists of a single SERTTA unit with half slotted and fully slotted elements facing the pin location, while the south side contains a half slotted graphite element. The rest of the lattice locations include standard fuel elements. Reflective boundary condition are imposed on all boundaries and greatly simplify the axial neutron streaming that is present in the configuration with full height and axial reflectors. Solutions from this simplified configuration are discussed in Section 6.3.3.

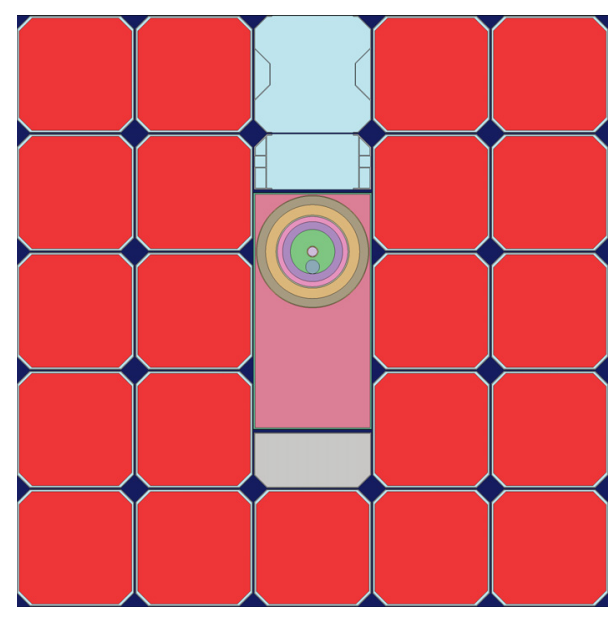

(a) $\mathrm{XY}$ view

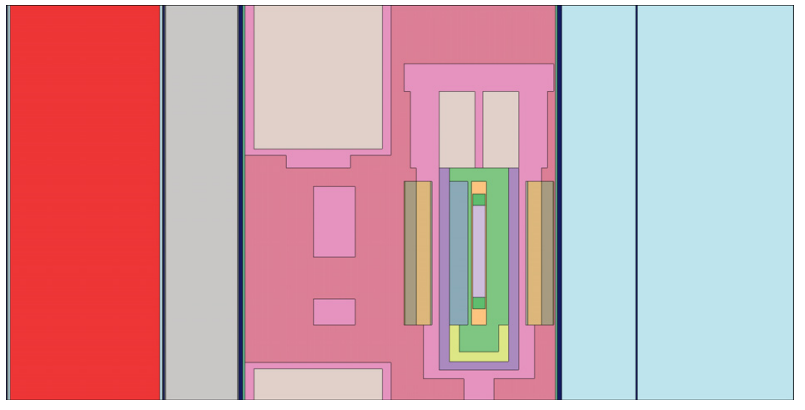

(b) YZ view

Figure 11: Serpent Model of the $5 \times 5$ Supercell with the SERTTA Unit

The Multi-SERTTA vehicle was added to the full core Serpent model, shown in Figure 12 , and comparison were made against a higher resolution MCNP model. The hodoscope penetration was later added to the model because it was found to significantly affect the power distribution in the core.

MAMMOTH uses Rattlesnake [34] as its neutron transport solver, which allows access to a variety of discretization methods for the transport equation. The system also includes the SPH equivalence method [35] and the Larson-Trahan method [36] to generate Tensor Diffusion Coefficients (TDCs). Serpent cannot generate physically meaningful diffusion coefficients in or near large neutron streaming regions like the air channels and hodoscope slot in TREAT. Therefore, TDCs are calculated with Rattlesnake for those regions. Finally, the errors introduced during the spatial homogenization and energy condensation processes are corrected via SPH in an effort to preserve the Monte Carlo reaction rates. 


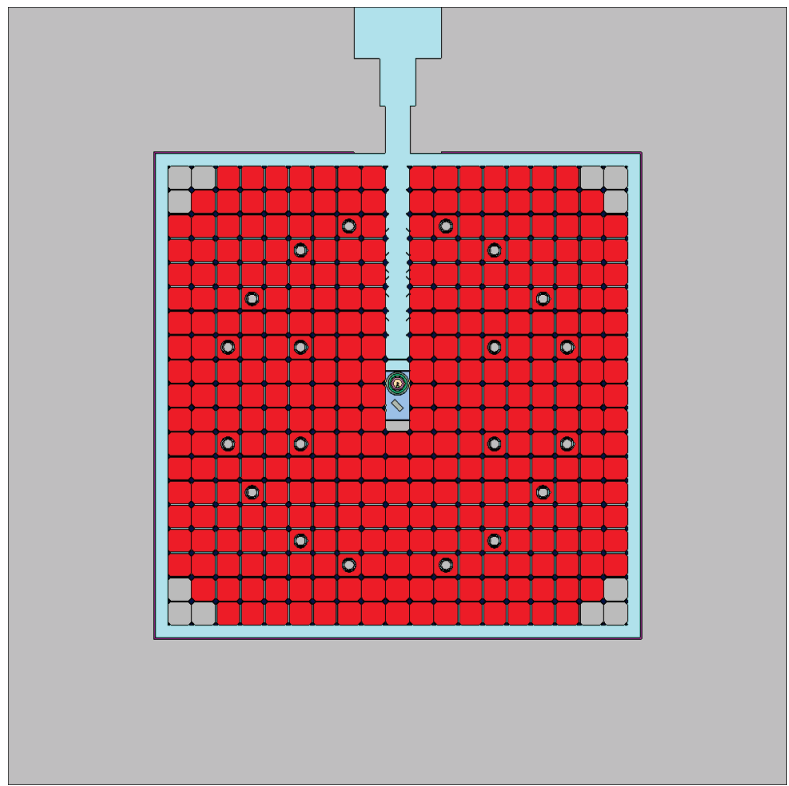

(a) $\mathrm{XY}$ view

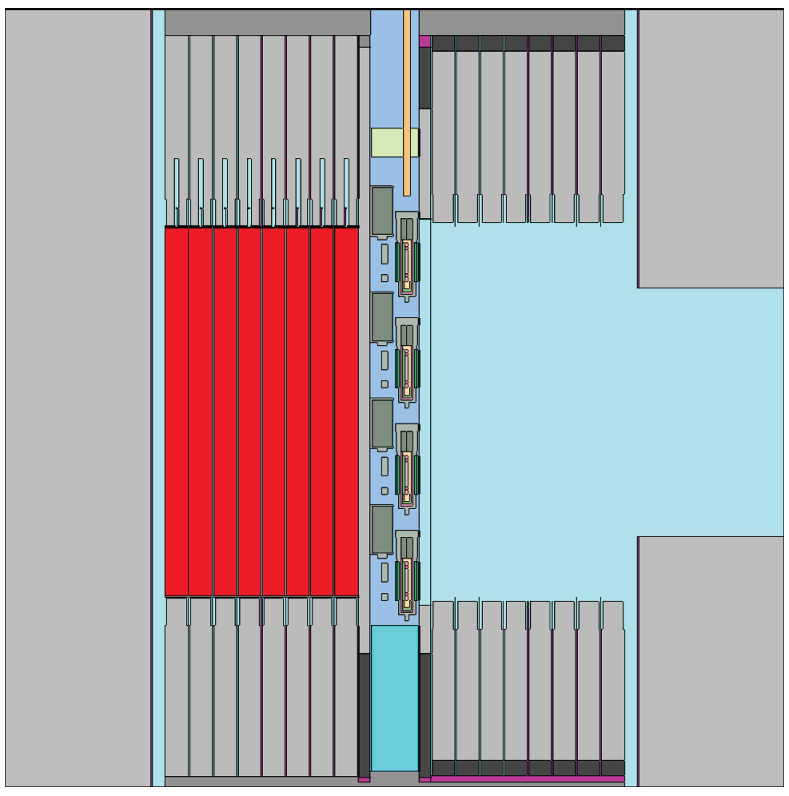

(b) YZ view

Figure 12: Full Core Serpent Model with the Multi-SERTTA Vehicle. 


\subsubsection{Mesh Development}

The mesh development for TREAT geometries is accomplished with a Python toolkit developed at INL that accesses the Cubit [37] application interface. In order to model the SERTTA unit, the python scripts were updated to allow a large number of concentric cylinders as well as the ability to create azimuthal regions within alternating cylinders. The full core models contain thousands of cross section regions, and therefore the correct assignment of cross sections becomes an arduous task in the input process. To circumvent this issue MAMMOTH employs an automatic mapping of cross sections using a material identification variable that is directly written into the mesh file. Both the radial and azimuthal regions in the pin as well as the material identification number are shown in Figure 13.

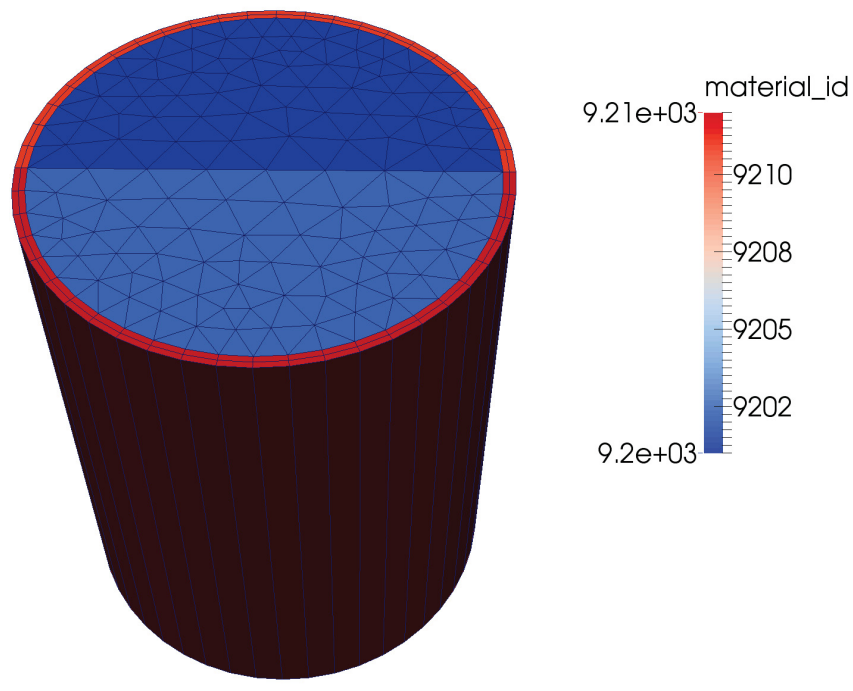

Figure 13: Pin Radial and Azimuthal Regions with Material Identification

The Multi-SERTTA vehicle requires a significant number of axial regions that propagate to the full core when an extruded mesh is used. In order to control the total number of elements in larger models a buffer region with tetrahedrons and pyramid elements is introduced which allows for very different level of resolution between the full core and the experiment region. Examples of the two mesh types are shown in Figure 14.

Future work includes the addition of the hodoscope penetration to the reflector region and the generation of the full core mesh with the Multi-SERTTA vehicle. 

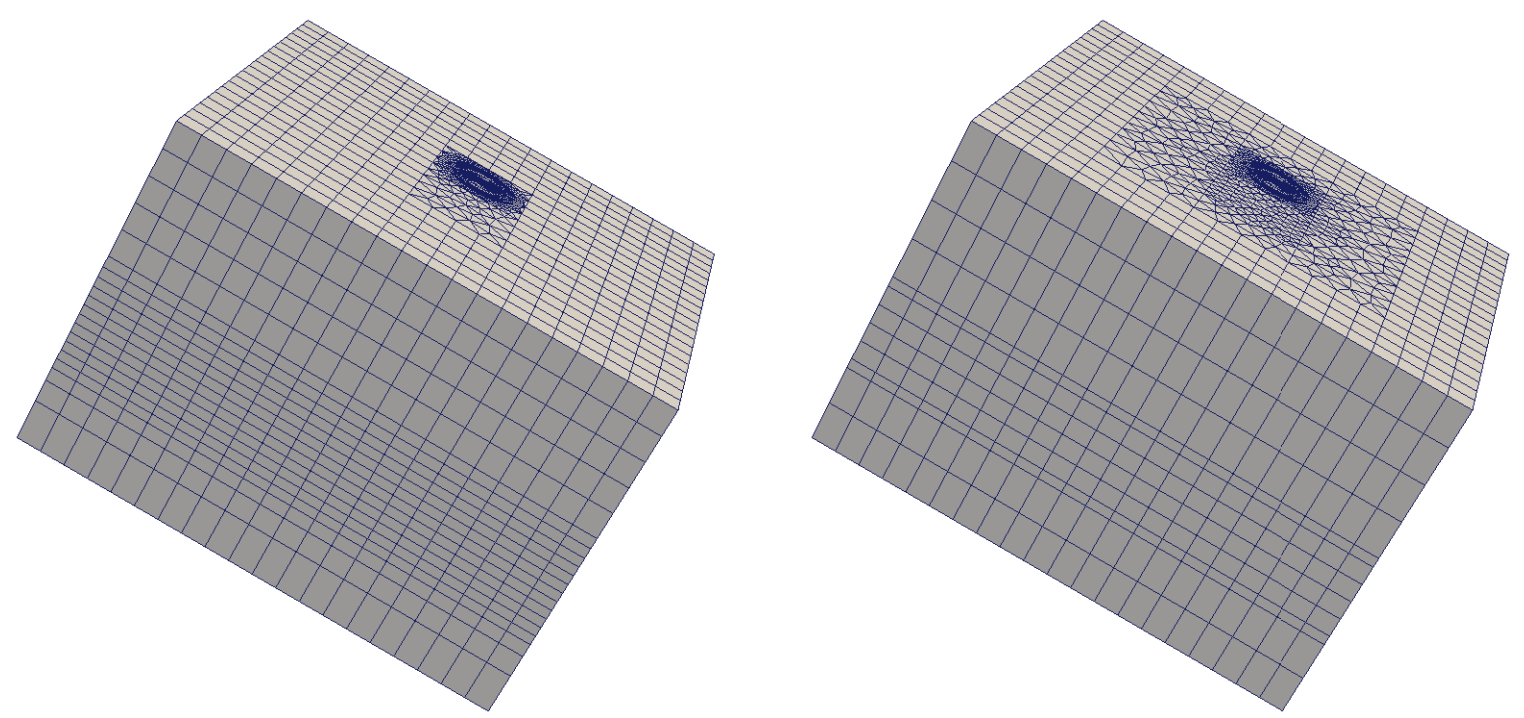

Figure 14: Extruded (left) and Hybrid (right) Meshes for the $5 \times 5$ Supercell

\subsubsection{Results}

The differences in the eigenvalues and integral parameters for the $5 \times 5$ SERTTA supercell are shown in Tables 1 and 2. The Serpent reference as well as Rattlesnake solutions with diffusion, SPH corrected diffusion $(\mathrm{SPH})$, discrete ordinates $\left(S_{12}\right)$, and TDC with SPH corrected diffusion (TDC-SPH) are included. The diffusion operator produces very large errors in the eigenvalue as well as the integral values. The $\sim 3.0 \%$ error in the integral values is consistent with the $\sim 3000 \mathrm{pcm}$ error. Note that the errors in the integral values for the source rate and absorption rate are the same, which is expected for a simulation without reflectors. The discrete ordinates solution is better, but without SPH equivalence cannot reproduce the Monte Carlo reference. Both the SPH and TDC-SPH appear to yield the same result and reduce the error from the diffusion calculation by a factor of $\sim 150$.

The pellet powers for the $5 \times 5$ SERTTA supercell, shown in Table 3 , confirm that the TDCSPH calculation produces the best results of all simulations, with the SPH a close second best. These results can be improved further by adding more cross section regions. Both natural uranium regions (top and bottom pellets) share the same cross sections. This is also the case for the enriched fuel pellets between the natural uranium regions, which explains the under-prediction at the bottom and the over-prediction at the top of the fuel stack, since the cross sections have been averaged over these regions. The enriched uranium pellet powers are predicted within $1.76 \%$ of the Monte Carlo reference with TDC-SPH corrected 
Table 1: Values of $k_{e f f}$ Calculated for the $5 \times 5$ SERTTA Supercell

\begin{tabular}{c|c|c} 
& keff & pcm difference \\
\hline Serpent & 1.08323 & $( \pm 2.1)$ \\
Diffusion & 1.05198 & -2885.1 \\
SPH & 1.08287 & -32.8 \\
$S_{12}$ & 1.08075 & -229.1 \\
TDC-SPH & 1.08287 & -32.9 \\
\hline
\end{tabular}

Table 2: Percentage Difference in Integral Parameters for the $5 \times 5$ SERTTA Supercell Relative to the Serpent Reference Solution

\begin{tabular}{c|c|c|c|c} 
& Diffusion & SPH & $S_{12}$ & TDC-SPH \\
\hline Absorption Rate & 2.96 & 0.02 & 0.22 & 0.02 \\
Flux & 1.90 & 0.01 & 0.09 & 0.01 \\
Source Rate & 2.96 & 0.02 & 0.22 & 0.02 \\
\hline
\end{tabular}

diffusion.

Table 3: Percentage Difference in Pellet Powers for the $5 \times 5$ SERTTA Supercell Relative to the Serpent Reference Solution

\begin{tabular}{l|c|c|c|c} 
& Diffusion & SPH & $S_{12}$ & TDC-SPH \\
\hline Nat U Pellet 1 & -0.11 & -3.00 & -3.28 & -2.45 \\
Enr U Pellet 1 & 11.67 & -2.01 & 4.47 & -1.76 \\
Enr U Pellet 2 & 11.64 & -1.27 & 3.32 & -1.02 \\
Enr U Pellet 3 & 12.42 & -0.37 & 4.42 & -0.16 \\
Enr U Pellet 4 & 12.25 & -0.43 & 4.38 & -0.29 \\
Enr U Pellet 5 & 13.18 & 0.43 & 5.20 & 0.47 \\
Enr U Pellet 6 & 13.13 & 0.38 & 4.96 & 0.28 \\
Enr U Pellet 7 & 14.39 & 1.40 & 5.66 & 1.11 \\
Enr U Pellet 8 & 15.74 & 1.99 & 7.81 & 1.42 \\
Nat U Pellet 2 & 5.88 & 3.46 & 1.61 & 2.85 \\
\hline
\end{tabular}

The fast and thermal flux distributions in the $5 \times 5$ supercell are depicted in Figure 15. The 
fast flux variation in this problem is not very large, but the thermal flux varies by an order of magnitude between the peripheral standard fuel assemblies and the experiment region. The presence of the slotted elements has a noticeable effect on the shape of the thermal flux around the experiment region. The slices shown in Figure 16 attest to the complexity of the model with significant flux gradients at various axial levels in the experiment region. The pin location becomes apparent as the source of fast neutrons in the experiment.

A close-up inspection of the thermal flux distribution inside the pressure vessel and fuel stack is included in Figure 17, which shows the asymmetry of the solution in the radial water reflector that surrounds the pin. The thermal flux has a peak that faces the slotted elements. The top and bottom of the stack experience a higher thermal flux level due to the presence of water reflector near those regions. A series of axial and radial slices of the pellet stack are depicted in Figures 18 to 20 that show detail of the flux distribution along the fuel stack and the radial distribution at the center of the fuel stack. Finally, the power density is shown in Figure 21. The power density is relatively constant through the fuel stack, with a clear peak facing the slotted elements. A diametric power density profile is included in Figure 22. 


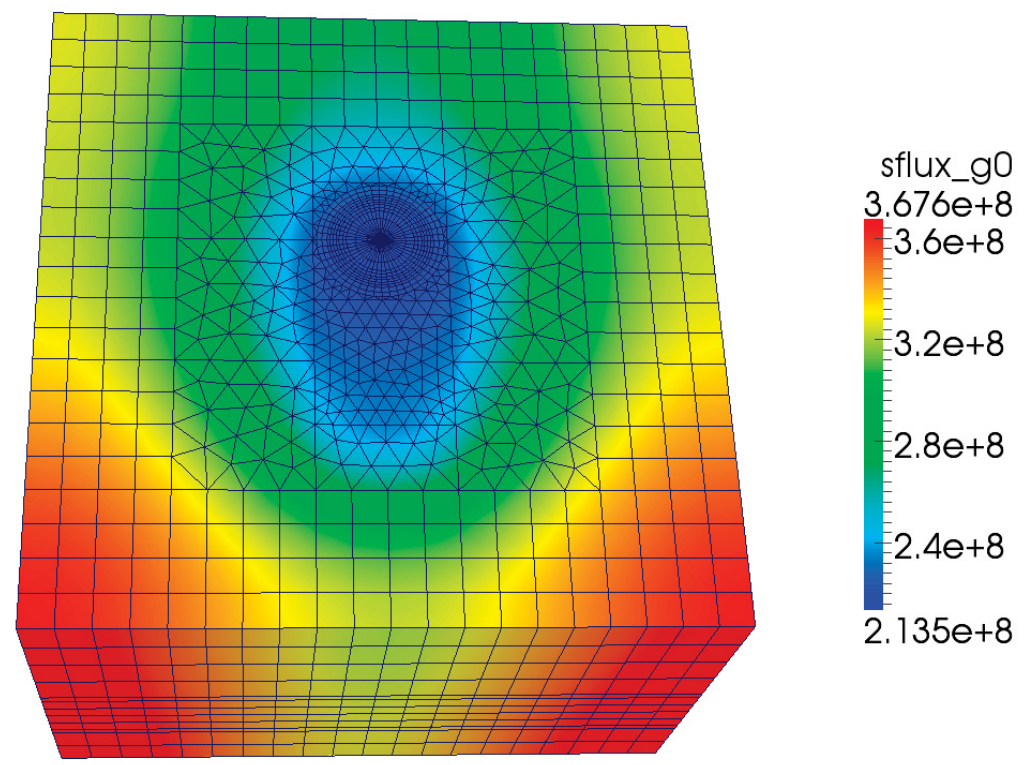

(a) Fast Flux (above $3.33 \times 10^{6} \mathrm{eV}$ )

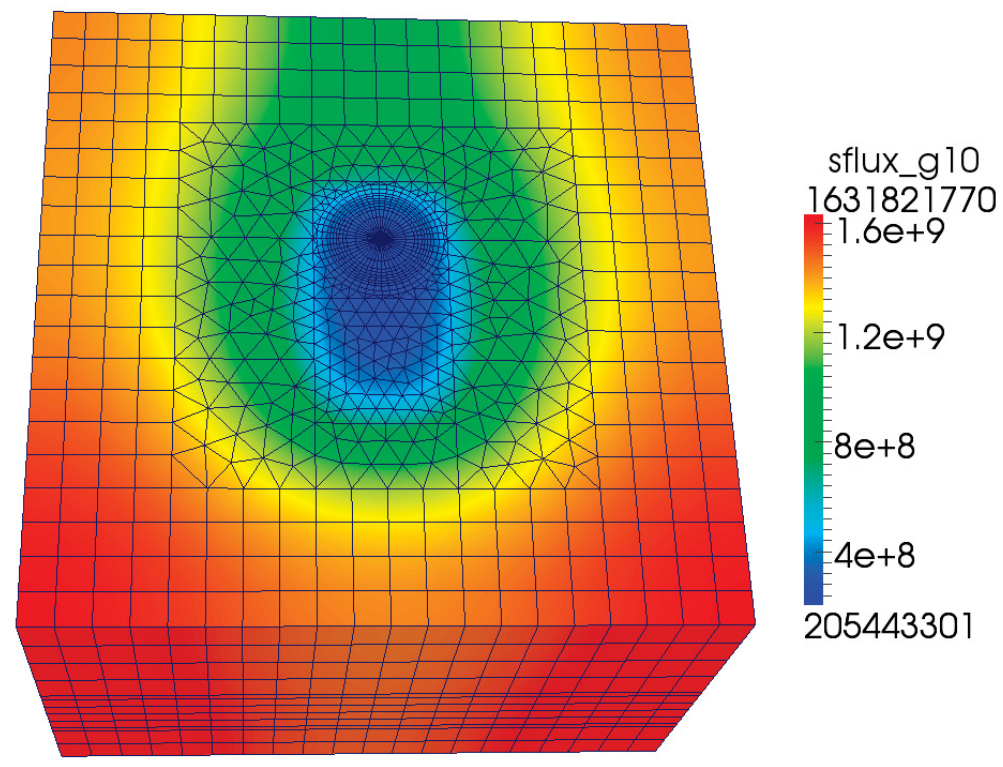

40

(b) Thermal Flux (below 2.00x $10^{-2} \mathrm{eV}$ )

Figure 15: Flux Distributions in the $5 \times 5$ SERTTA Supercell 


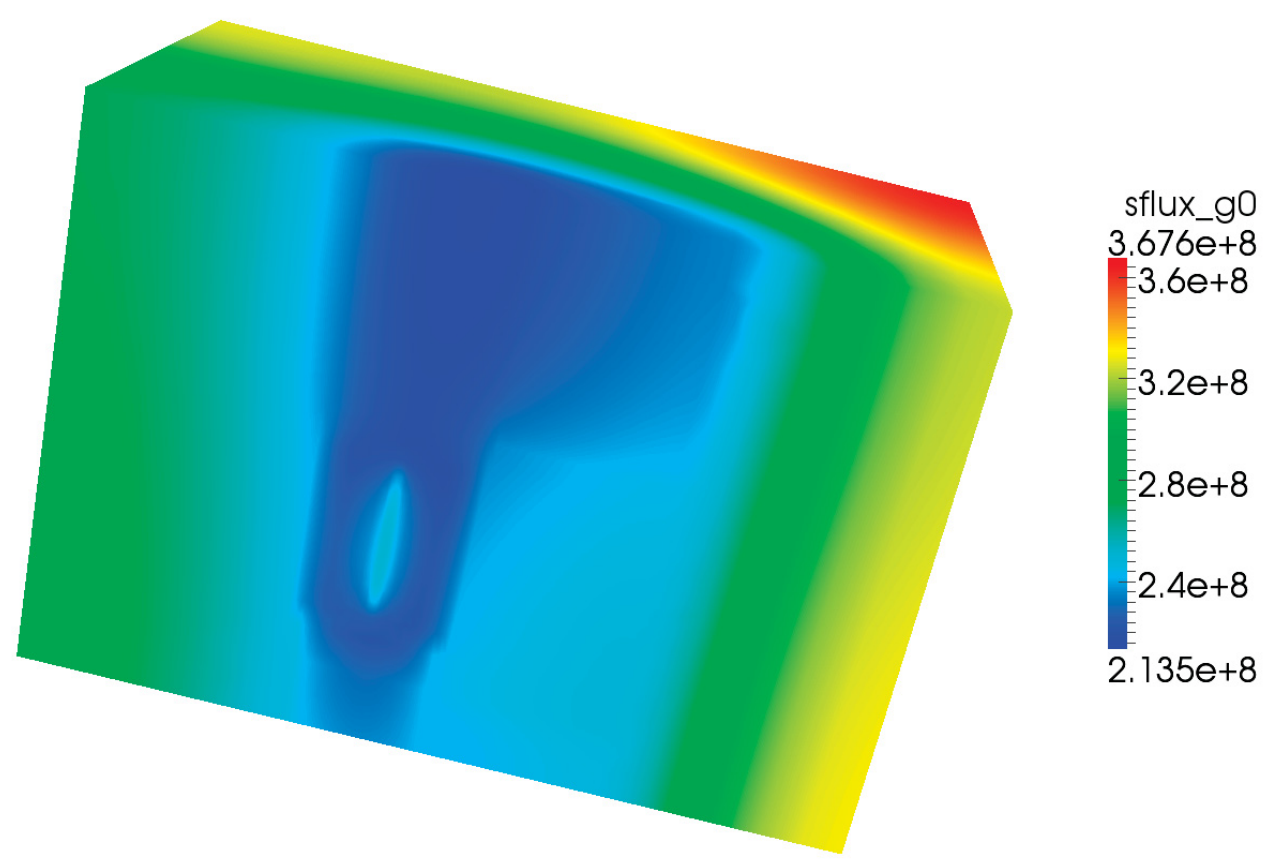

(a) Fast Flux (above $3.33 \times 10^{6} \mathrm{eV}$ )

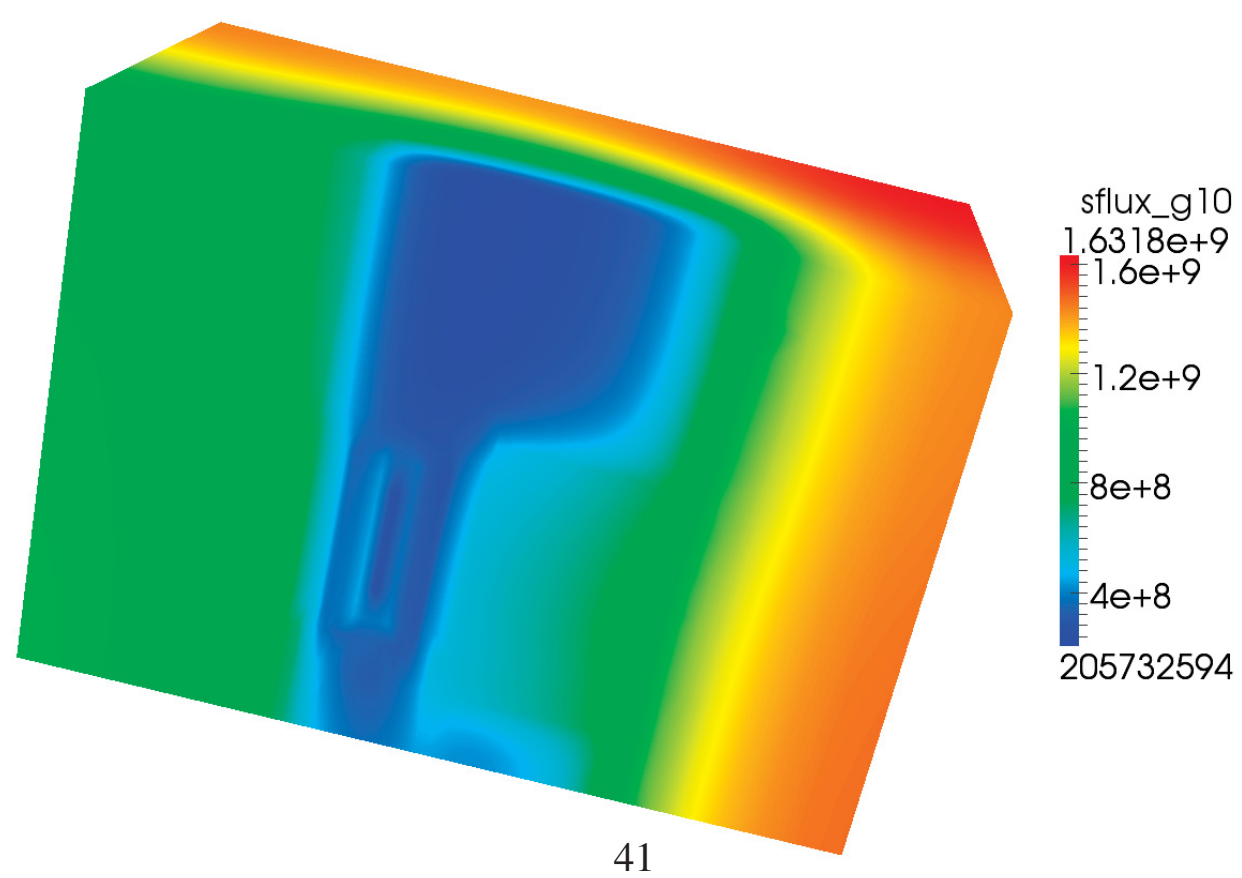

(b) Thermal Flux (below 2.00x10-2 eV)

Figure 16: Flux Distributions in a Slice of the $5 \times 5$ SERTTA Supercell 

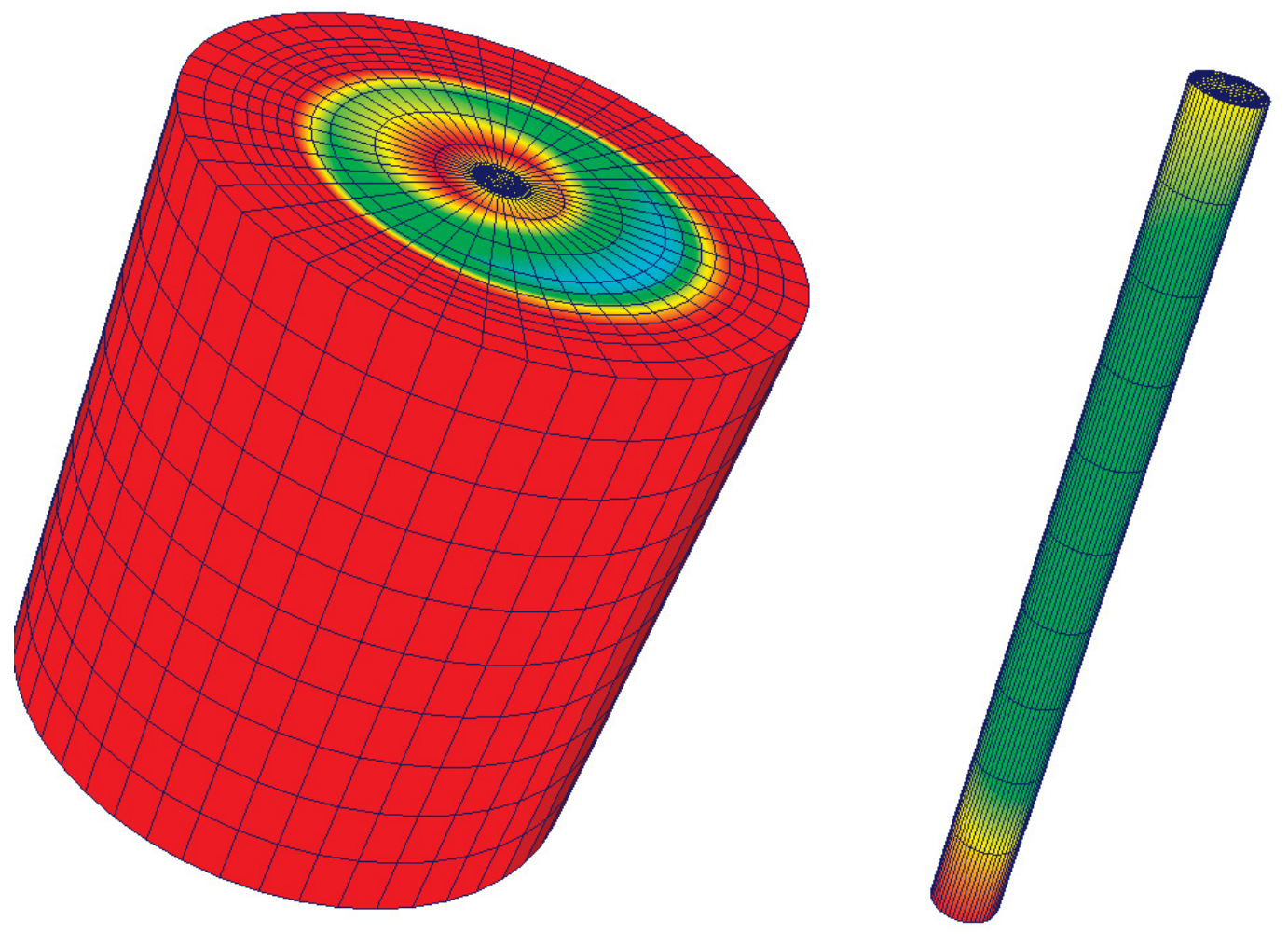

Figure 17: Thermal Neutron Flux (below 2.00 $\times 10^{-2} \mathrm{eV}$ ) inside the Pressure Vessel and Fuel Pin 

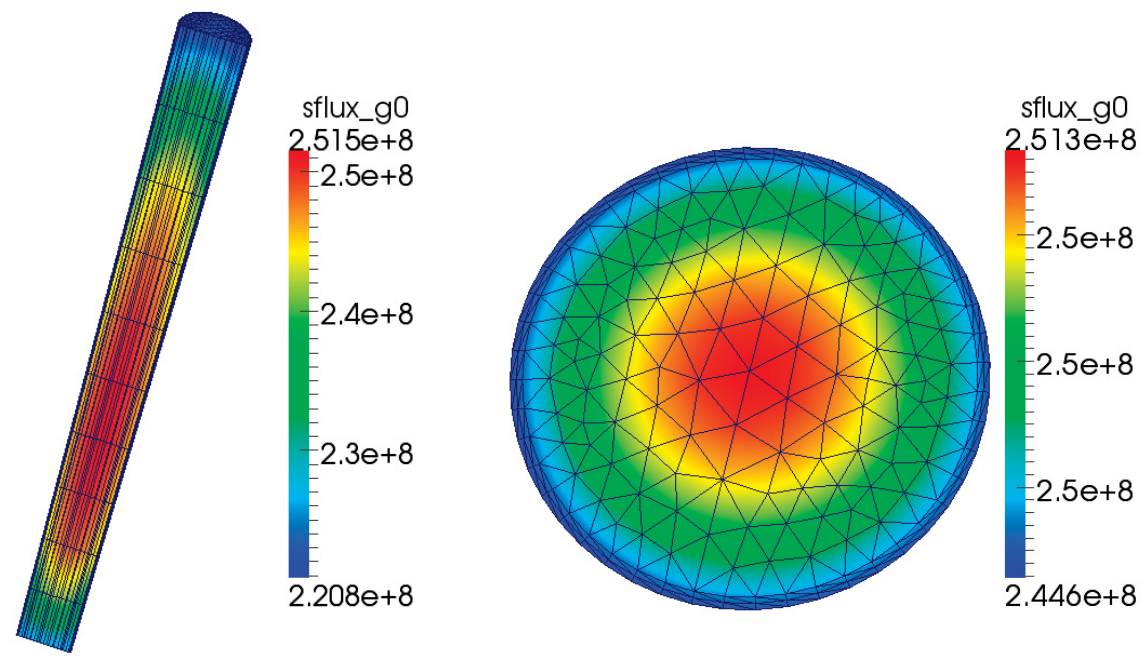

Figure 18: Fast Flux (above $3.33 \times 10^{6} \mathrm{eV}$ ) in the Fuel Pellet Stack (clip at axial centerline)
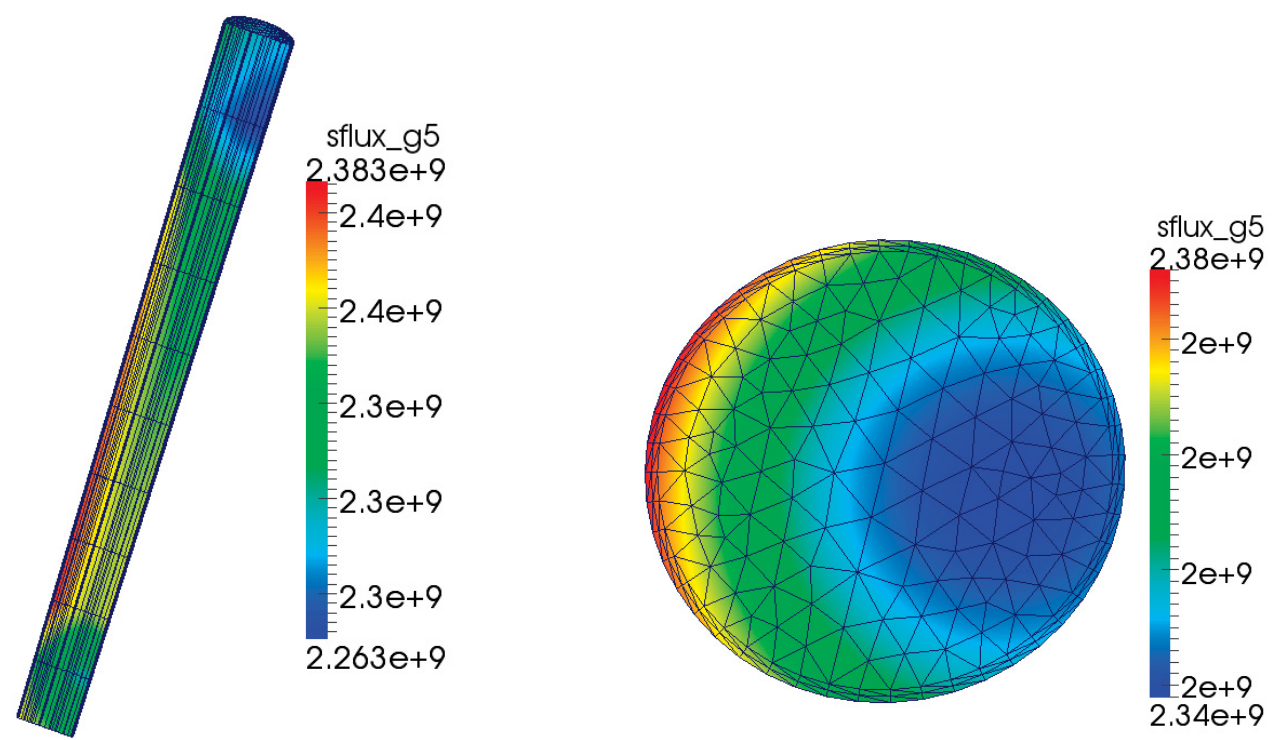

Figure 19: Thermal Flux (8.10 to $0.625 \mathrm{eV}$ ) in the Fuel Pellet Stack (clip at axial centerline) 

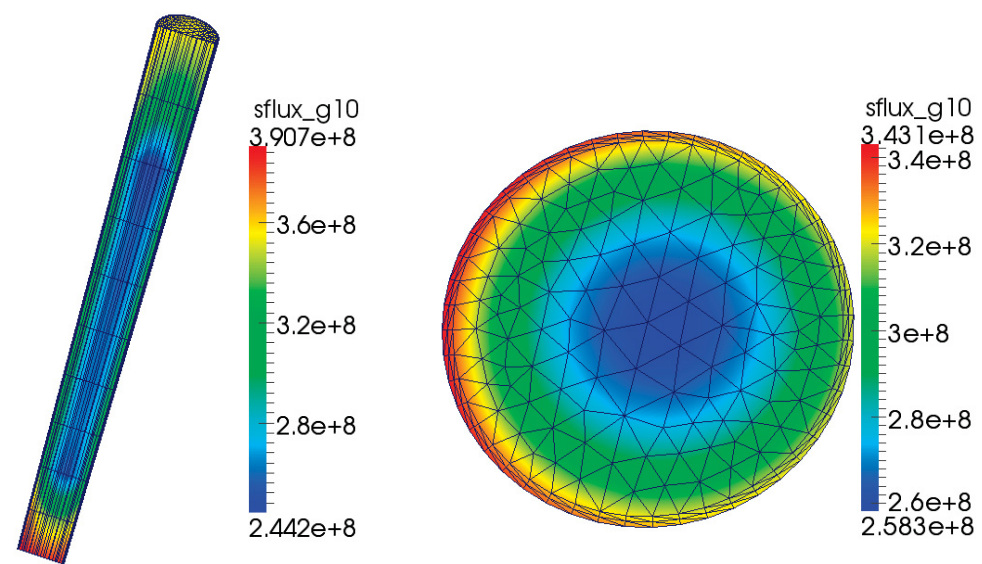

Figure 20: Thermal Flux (below 2.00 $\times 10^{-2} \mathrm{eV}$ ) in the Fuel Pellet Stack (clip at axial centerline)
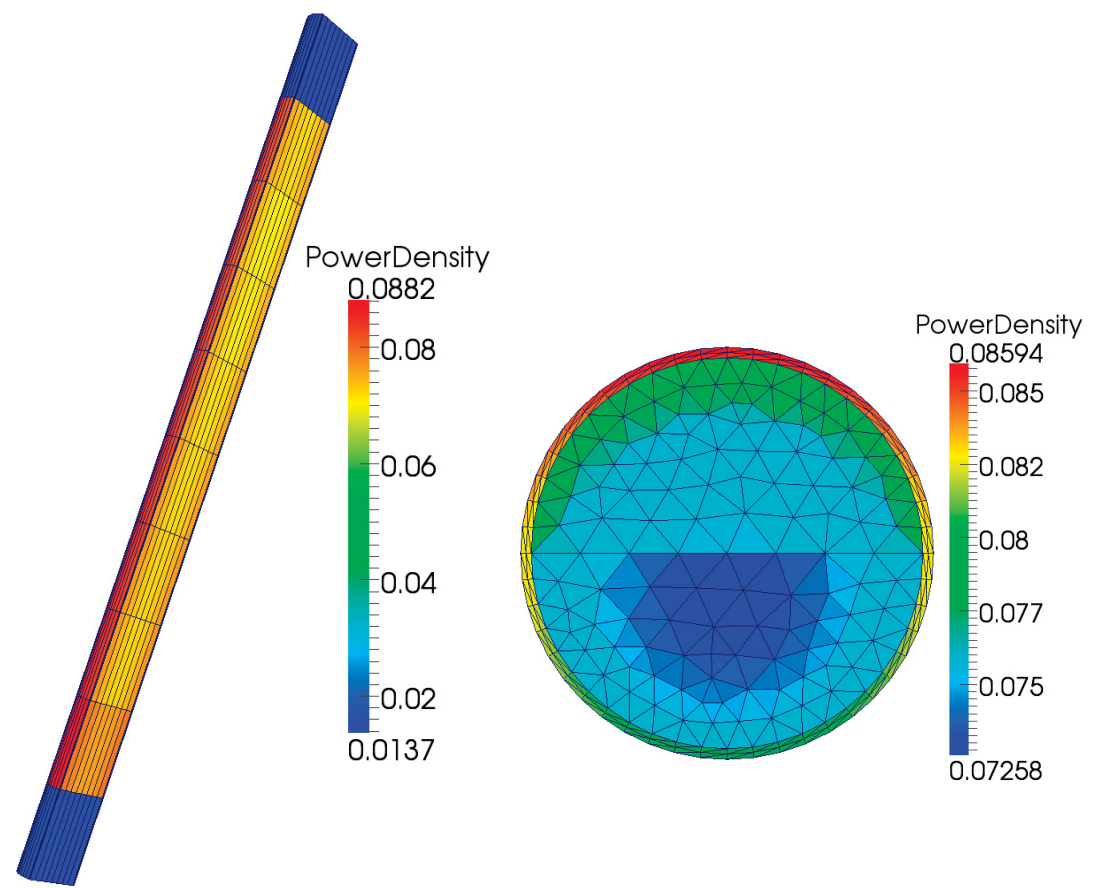

Figure 21: Power Density [W/cc] in the Fuel Pellet Stack (clip at axial centerline) 


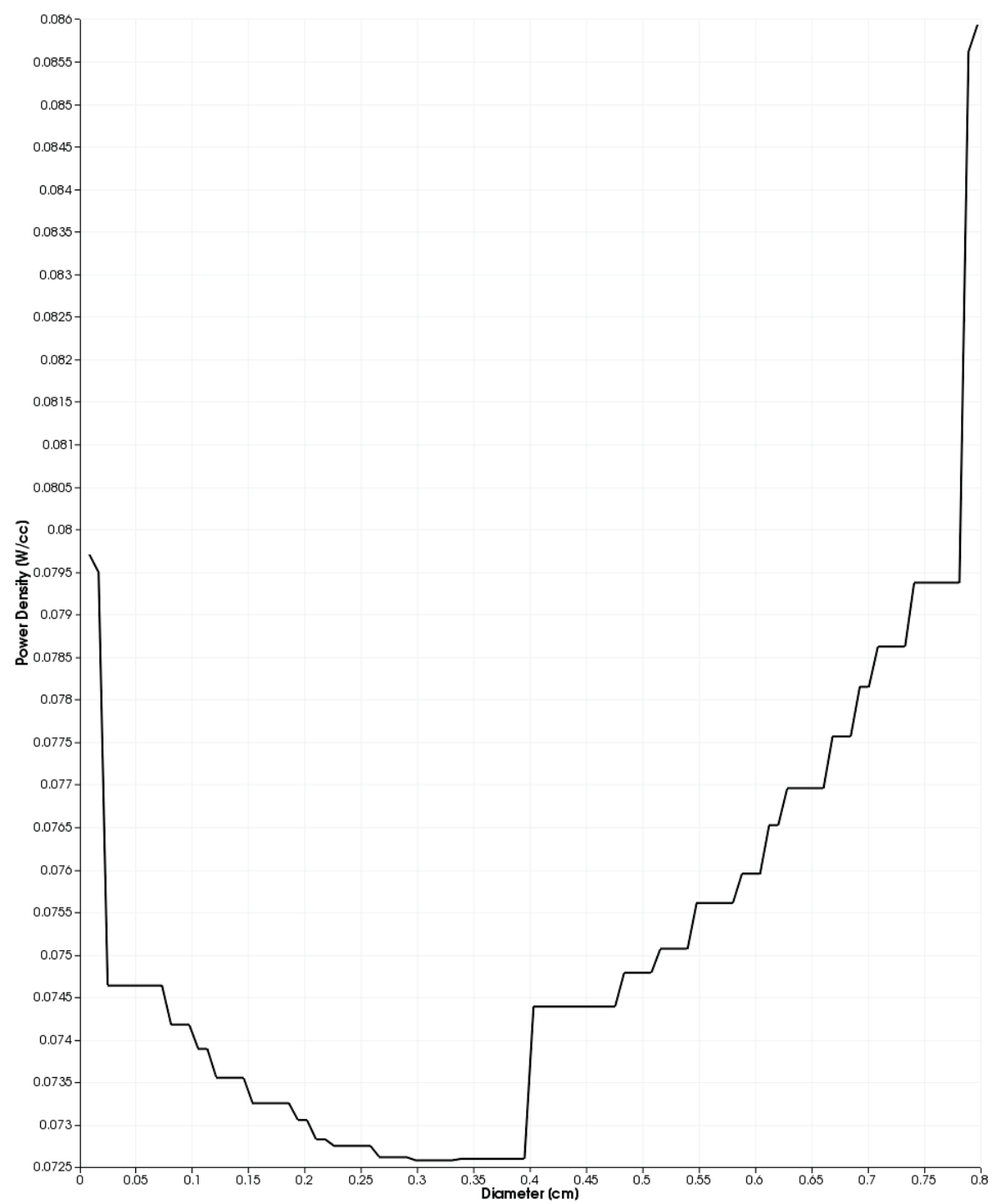

Figure 22: Diametric Power Density Profile at the Centerline (south to north) 


\subsubsection{Next Steps in Support of Transient Simulations}

High resolution modeling of the TREAT reactor with the Multi-SERTTA vehicle using MAMMOTH is underway. The work performed in late FY16 clearly demonstrates that MAMMOTH simulations are viable and can start producing valuable insights for experiment design by the end of FY17. Solutions for a smaller problem show that appropriately corrected diffusion calculations, with methods that reside within MAMMOTH, can deliver results that closely match reference Monte Carlo. The power in each enriched pellet of the SERTTA unit was predicted within $1.76 \%$ of the Monte Carlo reference.

Future work planned for FY17 includes the following extensions to the current status:

- Performing a full height $5 \times 5$ supercell calculation with the Multi-SERTTA vehicle,

- Adding hodoscope penetration to the meshing scripts and build a full core mesh,

- Preparing cross sections for the full core model at a fixed temperature,

- Checking the fidelity of the MAMMOTH full core steady state simulation against Serpent,

- Computing steady state coupling factors and compare against the reference,

- Building a full set of multi-dimensional cross section libraries,

- Checking the fidelity of the multi-dimensional cross sections against Serpent steady state results, and

- Running a transient simulation and computing the transient coupling factors. 


\section{Conclusions}

As TREAT restart approaches, many of the various partners supporting restart and operations, experiment design, instrumentation, benchmark development, and of course modeling and simulation are actively working together to develop testing plans to produce supporting data for current and future testing activities while supporting this core DOE activity. Advanced modeling and simulation efforts will effectively be refined and developed concurrently, enabling more integrated and advanced experiment design and testing. The first measurements in TREAT to support code validation are currently anticipated to begin in early 2017, five months into FY18. Experiment and instrumentation needs along with experiment positioning and procedures must be determined next within the next 12-14 months to be prepared for validation measurements. Additionally, ongoing and anticipated M\&S calculations and methods performed to support other restart activities (e.g., MultiSERTA, MPFD, etc.) along with reactivity computer software and interface, will need to be wrapped up in the same time period. While none of the MAMMOTH calculations will be considered to be fully qualified for safety purposes, successes in these calculations will make significant progress towards meeting validation needs.

Although it has been demonstrated that the various TREAT operations and support teams have been coordinated in their efforts in FY16, it should be noted that there is no formal organizational system in place to coordinate, drive and manage these important relationships. And while it seems logical that these efforts should become programmatically merged, the current approach, with the NEAMS modeling and simulation team working as a central hub to the various activities, was functionally successful in FY16. This informal but extremely important function is expected to grow signficantly over the next year as restart activities accelerate to successfully support near-term missions. However, current modeling and simulation efforts continue to take a long term view for effective deployment not only for TREAT, but also for other important multi-physics opportunities. 


\section{References}

[1] J. E. Kelly, S. A. Wright, V. Tikare, H. J. MacLean, E. J. Parma, Jr., C. D. Peters, M. E. Vernon, P. S. Pickard. Global nuclear energy partnership fuels transient testing at the Sandia National Laboratories nuclear facilities : planning and facility infrastructure options. Techical Report SAND2007-7308, Sandia National Laboratory, October 2007.

[2] L. Ramseth. Bringing a nuclear test reactor back to life at INL. Idaho Falls Post Register, October 2014.

[3] U.S. Department of Energy. Development of Light Water Reactor Fuels with Enhanced Accident Tolerance - Report to Congress.

[4] F. Kirn et al. Reactor Physics Measurements in TREAT. Technical Report ANL-6173, Argonne National Laboratory, 1960.

[5] J. Bess , M. D. DeHart. Baseline Assessment of TREAT for Modeling and Analysis Needs. Technical Report INL/EXT-15-35372, Idaho National Laboratory, 2015.

[6] B. Baker, J. Ortensi, M.D. DeHart, Y. Wang, F.N. Gleicher, and S. Schunert. Recommendations for TREAT Historical Data to Validate MAMMOTH. Technical Report INL/MIS-16-37647, Idaho National Laboratory, January 2016.

[7] F. N. Gleicher, J. Ortensi, et. al. The coupling of the neutron transport application RATTLESNAKE to the fuels performance application bison. In International Conference on Reactor Physics (PHYSOR 2014), Kyoto, Japan, May 2014.

[8] F. B. Brown et al. MCNP Version 5. Technical Report LA-UR-02-3935, Los Alamos National Laboratory, 2002.

[9] The RELAP5 Development Team. RELAP5/MOD3 Code Manual. Volumes 1 and 2 NUREG/CR-5535, INEL-95/0174, Idaho National Engineering Laboratory, August, 1995.

[10] ABAQUS. ABAQUS/CAE 6.11 User's Manual. Technical report, Dassault Systèmes., 2011.

[11] D. Gaston, C. Newman, G. Hansen, and D. Lebrun-Grand'e. Moose: A parallel computational framework for coupled systems of non-linear equations. Nucl. Eng. Design, 239(1768-1778), 2009. 
[12] T.L. Alberti, T.S. Palmer, J. Ortensi, and M.D. Dehart. Steady State Modeling of the Minimum Critical Core of the Transient Reactor Test Facility. In International Conference on Reactor Physics (PHYSOR 2016), Sun Valley, Idaho, USA, May 1 - 5 2016.

[13] J. Ortensi, M. D. DeHart, F. N. Gleicher, Y. Wang, S. Schunert, A. L. Alberti, T. S. Palmer. Full core TREAT kinetics demonstration using rattlesnake/BISON coupling within MAMMOTH. Technical Report INL/EXT-15-36268, Idaho National Laboratory, 2015.

[14] J. Ortensi, A. Alberti, Y. Wang, M.D. DeHart, F.N. Gleicher, S. Schunert, and T.S. Palrmer. Methodologies and requirements for the generation of physics data inputs to mammoth transient simulations in support of the transient reactor test facility. Technical Report NL/LTD-15-36265, Idaho National Laboratory, September 2015.

[15] T. H. Bauer W. R. Robinson. The M8 Power Calibration Experiment (M8CAL). Technical Report ANL-IFR-232, Argonne National Laboratory, May 1994.

[16] D. Andrs et al. RELAP-7 Level 2 Milestone Report: Demonstration of a Steady State Single Phase PWR Simulation with RELAP-7. Technical Report INL/EXT-12-25924, Idaho National Laboratory, 2012.

[17] M. Ellis, J. Ortensi, Y. Wang, K. Smith, and R. C. Martineau. Initial rattlesnake calculations of the hot zero power beavrs. Technical Report INL/EXT-13-30903, Idaho National Laboratory, Idaho Falls, ID, January 2014.

[18] R.L. Williamson et al. Multidimensional Multi-physics Simulation of Nuclear Fuel Behavior. Jou. Nucl. Mat., 423(149-163), 2012.

[19] F. N. Gleicher et al. The Application of MAMMOTH for a Detailed Tightly Coupled Fuel Pin Simulation with a Station Blackout. In Proceedings of Top Fuel 2016, Boise, ID, September 2016. American Nuclear Society.

[20] V. K. Patel, M. A. Reichenberger, J. A. Roberts, T. C. Unruh and D. S. McGregor. Simulated Performance of Micro-Pocket Fisison Detectors (MPFDs) in the Transient REAactor Test (TREAT) Facility using MCNP6, 2016.

[21] International handbook of evaluated reactor physics benchmark experiments, nea/nsc/doc(2006)1,. Technical Report NEA/NSC/DOC(2006)1, NEA No. 7258, 2015 Edition, OECD-NEA, Paris, March 2015. 
[22] D. W. Nigg and K. A. Steuhm, Eds. Advanced test reactor core modeling update project. Technical Report INL/EXT-12-27059, Idaho National Laboratory, Idaho Falls, ID, September 2012.

[23] N. E. Woolstenhulme, et al. Capabilities Development for Transient Testing of Advanced Nuclear Fuels at TREAT. In Proceedings of the Top Fuel 2016 Conference, Boise, ID, Sep 11-16 2016.

[24] et al. C. M. Hill. Mcnp water physics scoping study to support lwr accident tolerant fuel testing in treat. In PHYSOR 2016: Unifying Theory and Experiments in the 21st Century, Sun Valley, Idaho, USA, May 1 - 52016.

[25] et al. T. H. Bauer. Behavior of modern metallic fuel in treat transient overpower tests, 1990.

[26] B. C. Gneiting. Summary of report of hedl reference fuel treat tests,. Technical Report HEDL-TME 83-30, Hanford Engineering Development Laboratory, February 1984.

[27] G. Imel. Performance of hafnium and gadolinium self powered neutron detectors in the treat reactor,, 1996.

[28] M. Reichenberger et al. Advances in the Development and Testing of Micro-Pocket Fission Detectors (MPFDs). In IAEA International Conference on Research Reactors: Safe Management and Effective utilization, Vienna, Austria, 2015. International Atomic Energy Agency.

[29] T. Unruh, J. Rempe, D. McGregor, P. Ugorowski and M. Reichenberger. NEET Micro-Pocket Fission Detector - FY 2013 Status Report. Technical Report INL/EXT13-29346, Idaho National Laboratory, 2013.

[30] M. Reichenberger, T. Unruh, P. Ugorowski, T. Ito, J. Roberts, S. Stevenson, D. Nichols and D. McGregor. Micro-Pocket Fission Detectors (MPFDs) for In-Core Neutron Detection, 2016.

[31] C. Jensen, H. Ban, C. Folsom, R. O'Brien, T. Unruh, D. Wachs and N. Woolstenhulme. Instrumentation Development for Transient Testing in TREAT. In Proceedings of the 2015 ANS Winter Meeting, Las Vegas, NV, Nov. 6-10 2016 (to be published). American Nuclear Society.

[32] B. A. Baker, J. Ortensi, M. D. DeHart, Y. Wang, S. Schunert and F. N. Gleicher. Analysis Methods and Validation Activities for MAMMOTH Using M8 Calibration 
Series Data. Technical Report INL/EXT-16-40023, Idaho National Laboratory, Idaho Falls, ID, September 2016.

[33] J. Leppänen,. Development of a New Monte Carlo Reactor Physics Code. PhD thesis, Helsinki University of Technology, 2007.

[34] Y. Wang. Nonlinear diffusion acceleration for the multigroup transport equation discretized with sn and continuous fem with rattlesnake. In Proceedings to the International Conference on Mathematics, Computational Methods \& Reactor Physics (M\&C 2013), Sun Valley, Idaho, USA, May 2013.

[35] A. Hébert, G. Mathonniere. Development of a third generation superhomogenization method for the homogenization of pressurized water reactor assembly. Nuc. Sci. Eng., $2: 115,1993$.

[36] T.J. Trahan. An Asymptotic, Homogenized, Anisotropic, Multigroup Diffusion Approximation to the Neutron Transport Equation. PhD thesis, University of Michigan, 2014.

[37] Randy Morris. Cubit 15.0 user documentation. Technical report, ETI, UT, 2014. 